\title{
Mass flux of dispersed particles in turbulence: Representations and the influence of correlation structure in gravitational settling
}

\author{
C.P. Stafford \\ School of Engineering, Newcastle University, Newcastle upon Tyne NE1 7RU, UK \\ D.C. Swailes* \\ School of Mathematics, Statistics \& Physics, Newcastle University, Newcastle upon Tyne NE1 7RU, UK
}

(Dated: April 19, 2021)

\begin{abstract}
Different integral representations for the mass flux of inertial particles transported by turbulent gas flows have been proposed. These are discussed and analysed. Each formulation provides its own insights into the underlying physical processes governing the resulting flux. However none of the representations, as it stands, provides an explicit closed-form expression in terms of known statistical properties of the flow and parameters governing particle dynamics. We consider the representations in terms of their potential for reduction to closed-form models. To enable an analysis uncomplicated by the presence of many coupled interactions we confine our attention to the classic test case of mono-disperse particles in homogeneous, isotropic turbulent flows, and subject to a uniform gravitational field. The modification of the mean particle settling velocity resulting from their preferential sampling of fluid velocities is captured by the flux representations. A distributionbased symmetry analysis coupled with a correlation splitting technique is used to reduce and simplify the terms appearing in the flux integrals. This prompts a strategy for closure modelling of the resulting expressions in terms of correlations between the sampled fluid velocity and fluid strainrate fields. Results from particle-trajectory based simulations are presented to assess the potential of this closure strategy.
\end{abstract}

PACS numbers: 47.55.Kf, 47.27.De, 05.10.Gg

Keywords: disperse multiphase flow, turbulence, pdf kinetic equations, continuum equations

\section{INTRODUCTION}

Particle-number and particle-velocity distributions, combined with statistical characterisations of turbulence, play an important role in the study of particle transport in turbulent flow. Equations governing the behaviour of such distributions, or statistical moments derived from these, provide a basis for exploring the physical behaviour of disperse multiphase flows. In this context the notion of the particle mass flux is fundamental; it is central to the formulation of mass and momentum conservation equations.

To elaborate, consider inertial particles transported in a turbulent flow with velocity field $\mathbf{u}(\mathbf{x}, t)$. The current work assumes that the particle density is much higher ( $>10^{3}$ times) than that of the fluid, as is typical of gas-solid flows. Furthermore, the focus is on dilute systems in which hydrodynamic interactions and interparticle collisions are considered negligible, as is applicable in the regime of a low particle volume fraction. Denote the position and velocity of an individual particle in the flow at time $t$ by $\boldsymbol{x}(t)$ and $\boldsymbol{v}(t)$. Within the framework of a statistical description of turbulence the velocity field $\mathbf{u}$ is treated as stochastic, and the particle mass flux, and other properties, are defined in terms of ensemble averages $\langle\cdot\rangle$ over realisations of this field and the initial state $\boldsymbol{z}^{0}=(\boldsymbol{x}(0), \boldsymbol{v}(0))$ : In particular,

\footnotetext{
*d.c.swailes@newcastle.ac.uk
}

the particle mass flux (strictly, the particle number density flux) is $\langle\varrho(\mathbf{x}, t) \boldsymbol{v}(t)\rangle$, where $\varrho(\mathbf{x}, t)=\delta(\boldsymbol{x}(t)-\mathbf{x})$ is the fine-grain (single realisation) probability density function (pdf) for the particle position. This flux can be expressed in terms of the ensemble-averaged number density $\rho(\mathbf{x}, t)=\langle\varrho(\mathbf{x}, t)\rangle$ and the mean particle velocity field $\overline{\mathbf{v}}(\mathbf{x}, t)$,

$$
\langle\varrho(\mathbf{x}, t) \boldsymbol{v}(t)\rangle=\rho(\mathbf{x}, t)\langle\boldsymbol{v}(t)\rangle_{\mathbf{x}}=\rho(\mathbf{x}, t) \overline{\mathbf{v}}(\mathbf{x}, t) .
$$

Here $\langle\boldsymbol{v}\rangle_{\mathbf{x}}=\overline{\mathbf{v}}$ denotes a conditional average, based on the sub-ensemble of realisations such that $\boldsymbol{x}(t)=\mathbf{x}$. The continuity (number conservation) equation for the particle phase follows as

$$
\partial_{t} \rho=\langle\dot{\varrho}\rangle=\langle-\boldsymbol{v} \cdot \boldsymbol{\partial} \varrho\rangle=-\boldsymbol{\partial} \cdot \rho \overline{\mathbf{v}}
$$

where $\partial_{t}=\partial / \partial t$ and, in a Cartesian co-ordinate frame, $\boldsymbol{\partial}=\left(\partial_{1}, \partial_{2}, \partial_{3}\right), \partial_{j}=\partial / \partial x_{j}$.

The aim is to derive representations for the mass flux $\rho \overline{\mathbf{v}}$ appearing in (1) in the form

$$
\rho \overline{\mathbf{v}}=\rho \boldsymbol{d}-\boldsymbol{D} \cdot \boldsymbol{\partial} \rho
$$

where the convective (drift) velocity $\boldsymbol{d}$ and the gradient diffusion tensor $\boldsymbol{D}$ are given explicitly in terms of known statistical properties of $\mathbf{u}$. This challenge has been addressed by a number of authors who, using quite distinct methods, have developed alternative representations for these convective and diffusive contributions to the mass flux. Whilst the representations that emerge from these different approaches are distinct, they do share common 
features. Most notably, all require closure modelling to obtain explicit expressions. We compare and contrast these different flux representations, both in terms of the limits of their applicability as well as the potential for closure analysis that they afford.

To permit precise and detailed analysis, and following the seminal work by Maxey [1], we consider the test case of mono-disperse particles settling under gravity in a statistically stationary, homogeneous and isotropic flow. This allows for a uniform particle concentration, $\rho$, generating a purely convective mass flux; Eq. (2) reduces to $\overline{\mathbf{v}}=\boldsymbol{d}$, so that any model for $\boldsymbol{d}$ can be assessed directly via the evaluation of $\overline{\mathbf{v}}$ obtained from particle trajectory based simulations.

Maxey's work showed that the average settling rate of particles in turbulence can be greater than that in stationary fluid. The physical mechanism for this enhanced settling is attributable to the preferential sweeping of particles, which originates due to a bias in the sampling of downward regions of fluid flow. For small particles this manifestation notably occurs through the preferential concentration phenomenology, in which the turbulent structures play a key role in the settling enhancement due to the associated bias in sampling of highstrain, low-vorticity regions in the flow field by particles. Flux representations that are amenable to closure models based on these physical features of the system are therefore crucial, and are the subject of this paper.

Particle drift has also previously been investigated in detail in the context of particle pair clustering [2, 3], in which theories for the different mechanisms leading to a spatial accumulation of particles were contrasted. Additionally, consideration of particle drift from a PDF perspective has previously been used to analyse gravitational settling, but only in the case of dense suspensions where the majority of the increase in settling velocity is attributed to the higher particle volume fraction [4]. Modelling of the increase in settling velocity which occurs due to the preferential sweeping by turbulence has not been attempted using PDF methods before, and it is this which the present study aims to address.

Investigation into the dependence of particle clustering on mechanisms that act outside of the dissipation range of turbulence has identified path history effects as being a key factor across all scales $[2,5]$. The effect of trajectory history on clustering has also been studied via the use of the deformation tensor which represents the Eulerian-Lagrangian transformation along a trajectory, both to evaluate the moments of the particle number density [6-8], and further through the use of dynamical systems approaches in which the Lyapunov exponents of particles are used to quantify the rate of contraction and compressibility of the particle velocity field $[9,10]$.

Section II summarises the various approaches, and resulting flux representations, that have been developed. Section III presents a distribution-based symmetry analysis; this not only reduces the complexity of derived flux expressions, but also highlights important features of sta- tistical correlations that appear within these. An approach to developing closure models is proposed in Section IV. We make use of stochastic, particle-tracking simulations to assess the efficacy of both the underlying flux representations and the associated closure strategy. Results from these simulation-based assessments are presented in Section V.

\section{FLUX REPRESENTATIONS}

A number of methodologies have been introduced for constructing representations to $\rho \overline{\mathbf{v}}$. These can be divided into two categories, depending on whether a particle momentum equation or a particle velocity field is introduced. The first approach considers momentum transport [11]

$$
\partial_{t}\langle\varrho \boldsymbol{v}\rangle=\langle\dot{\varrho} \boldsymbol{v}+\varrho \dot{\boldsymbol{v}}\rangle=-\boldsymbol{\partial} \cdot \rho \overline{\mathbf{v} \mathbf{v}}+\langle\varrho \mathbf{F}\rangle,
$$

where $\mathbf{F}$ governs particle dynamics through the equation of motion

$$
\ddot{\boldsymbol{x}}(t)=\dot{\boldsymbol{v}}(t)=\mathbf{F}(\boldsymbol{x}, \boldsymbol{v} ; \mathbf{u}) .
$$

The majority of works focus on the classic linear drag model

$$
\mathbf{F}(\mathbf{x}, \mathbf{v} ; \mathbf{u})=\tau^{-1}(\mathbf{u}(\mathbf{x}, t)-\mathbf{v})+\mathbf{g}
$$

where $\tau^{-1}$ is the particle response rate, and $\mathbf{g}$ denotes gravitational acceleration. In this work we are concerned solely with systems that exhibit statistically stationary and spatially uniform states in homogeneous turbulence, $\langle\mathbf{u}\rangle=\mathbf{0}$. In such cases Eqs. (3), (5) give

$$
\rho \overline{\mathbf{v}}=\rho \mathbf{v}_{g}+\langle\varrho \mathbf{u}\rangle,
$$

where $\mathbf{v}_{g}=\tau \mathbf{g}$ denotes the gravitational settling velocity. Any deviation of the mean particle settling velocity $\overline{\mathbf{v}}$ from $\mathbf{v}_{g}$ is therefore a result of the preferential sampling of the fluid velocity $\langle\varrho \mathbf{u}\rangle$. The challenge is to formulate closures for this quantity, and to gain insight into the physical mechanisms underlying the mass flux contribution emerging from this term. In passing we note that this term is also central to the study of inhomogeneous systems [12].

One approach to closing this drift flux term is that of functional correlation splitting [13]. In the present context this leads to the approximation $\langle\varrho \mathbf{u}\rangle \approx \rho \overline{\boldsymbol{\kappa}}$, with $[14$ $17]$

$$
\bar{\kappa}_{i}=\int_{0}^{t}\left\langle\mathcal{H}_{k j}\left(t ; t^{\prime}\right) \partial_{k} \mathrm{R}_{j i}\left(\boldsymbol{r}, t^{\prime}-t\right)\right\rangle_{\mathbf{x}} d t^{\prime}
$$

In Eq. (7), $\boldsymbol{r}=\boldsymbol{x}\left(t^{\prime}\right)-\mathbf{x} ; \mathbf{R}$ is the two-point, two-time correlation tensor for the homogeneous field $\mathbf{u}$,

$$
\mathbf{R}\left(\mathbf{x}^{\prime}-\mathbf{x}, t^{\prime}-t\right)=\left\langle\mathbf{u}\left(\mathbf{x}^{\prime}, t^{\prime}\right) \mathbf{u}(\mathbf{x}, t)\right\rangle,
$$


and $\mathcal{H}\left(t ; t^{\prime}\right)$ is the particle response tensor. From Eqs. (4) and (5) the differential system for $\mathcal{H}$ is

$$
\ddot{\mathcal{H}}=-\tau^{-1}(\dot{\mathcal{H}}-\Gamma \cdot \mathcal{H})
$$

with initial $\left(t=t^{\prime}\right)$ conditions, $\mathcal{H}=\mathbf{O}, \dot{\mathcal{H}}=\mathbf{I}$. The particle response tensor has the physical interpretation of describing the effect of a perturbation in the fluid velocity field on the particle trajectory at subsequent times. In Eq. (8) $\Gamma_{i m}(t)=\partial_{m} \mathrm{u}_{i}(\boldsymbol{x}(t), t)$. The dependence of $\mathcal{H}$ on these fluid velocity gradients sampled along particle trajectories is a crucial feature of this representation, and it is through $\boldsymbol{\Gamma}$ that the effect of gravity is implicit in Eq. (8). This is in accordance with the interpretation of enhanced drift being attributable to inhomogeneities in the instantaneous distribution of particles, with preferential sampling of regions of high strain over those of high vorticity. We return to this aspect of the representation, and its implication for closure, in Section IV.

That $\overline{\boldsymbol{\kappa}}$ provides a description for the settling enhancement of inertial particles which is consistent with the mechanism of preferential sweeping can be seen in the expression $\langle\varrho \mathbf{u}\rangle$ which is being modelled; a bias in the sampling of downward regions of flow will be captured within this average, and therefore also in Eq. (7). The average $\left\langle\mathcal{H}^{\top}: \boldsymbol{\partial R}\right\rangle$ appearing in $\overline{\boldsymbol{\kappa}}$ can be interpreted as the force per unit mass that particles experience at a given point in time as a result of fluid turbulence, with the accumulation of these acceleration contributions over the trajectory history resulting in the drift velocity described by $\overline{\boldsymbol{\kappa}}$. This can be further elucidated by considering the definition of the response tensor $\mathcal{H}$ as a functional derivative $[15,18]$; it is then possible to express the average $\left\langle\mathcal{H}^{\top}: \boldsymbol{\partial R}\right\rangle$ in the form

$$
\left\langle\frac{\delta}{\delta \mathbf{u}\left(\boldsymbol{x}\left(t^{\prime}\right), t^{\prime}\right) \delta t^{\prime}} \cdot \mathbf{R}\left(\boldsymbol{r}, t^{\prime}-t\right)\right\rangle
$$

This representation provides the interpretation of $\left\langle\mathcal{H}^{\top}\right.$ : $\partial \mathbf{R}\rangle$ as the average response in the fluid correlation tensor $\mathbf{R}$ as sampled by particles that results from a perturbation in the flow field $\mathbf{u}$ at an earlier time. This makes it clear that it is the flow correlations experienced by particles as a result of turbulence at all scales within the flow that are responsible for the settling velocity increase, and therefore the description (7) provided by $\overline{\boldsymbol{\kappa}}$ offers a generalisation of the centrifuging mechanism [1].

Another important feature of this correlation splitting approach is that it provides exact representations when the underlying flow field $\mathbf{u}$ is Gaussian. This allows for rigorous testing of closure models; the influence of nonGaussian features can either be assessed or eliminated. It is however possible to extend the approach to account for the non-Gaussian aspects of the flow; this is discussed further in Section V A. The inclusion of higher order nonGaussian correlations within the particle phase is also illustrated in the closure analysis presented in Section IV.
The second approach to constructing representations for the flux $\rho \overline{\mathbf{v}}$ invokes the concept of a particle velocity field. This is a subsidiary, stochastic field $\mathbf{V}(\mathbf{x}, t)$ associated with each realization of $\mathbf{u}$, such that the corresponding particle density $\varphi(\mathbf{x}, t)=\langle\varrho\rangle_{\mathbf{u}}^{z^{0}}$, the ensemble over initial states for a given realisation of $\mathbf{u}$, satisfies

$$
\partial_{t} \varphi+\boldsymbol{\partial} \cdot \varphi \mathbf{V}=0
$$

It follows that

$$
\varphi(\mathbf{x}, t)=\rho^{0}\left(\mathbf{y}^{0}\right) \exp \left[-\int_{0}^{t} \boldsymbol{\partial} \cdot \mathbf{V}\left(\boldsymbol{y}^{\prime}, t^{\prime}\right) d t^{\prime}\right] .
$$

Here $\rho^{0}$ defines the distribution of the initial particle positions, and $\boldsymbol{y}^{\prime}=\boldsymbol{y}\left(t^{\prime} ; \mathbf{x}, t\right)$ is the solution to

$$
\frac{d \boldsymbol{y}^{\prime}}{d t^{\prime}}=\mathbf{V}\left(\boldsymbol{y}^{\prime}, t^{\prime}\right)
$$

satisfying $\boldsymbol{y}(t ; \mathbf{x}, t)=\mathbf{x}$. In Eq. (10), $\mathbf{y}^{0}=\boldsymbol{y}(0 ; \mathbf{x}, t)$. From this the mass flux $\rho \overline{\mathbf{v}}=\langle\varphi \mathbf{V}\rangle^{\mathbf{u}}$ can be written as

$$
\rho \overline{\mathbf{v}}=\left\langle\rho^{0}\left(\mathbf{y}^{0}\right) \mathbf{V}(\mathbf{x}, t) \exp \left[-\int_{0}^{t} \boldsymbol{\partial} \cdot \mathbf{V}\left(\boldsymbol{y}^{\prime}, t^{\prime}\right) d t^{\prime}\right]\right\rangle .
$$

Here the ensemble average is over all realisations of $\mathbf{u}$, with $\boldsymbol{y}^{\prime}$ defined accordingly in each.

A number of approaches for specifying $\mathbf{V}$ have been developed: Maxey [1] constructed $\mathbf{V}$ based on expansion of the particle equation of motion (4), (5) in terms of $\tau$. For small Stokes number $S t=\tau / \tau_{f}$, where $\tau_{f}$ is a characteristic fluid time scale, this leads to

$$
\mathbf{V}=\mathbf{u}+\mathbf{v}_{g}-\tau\left(\partial_{t} \mathbf{u}+\left(\mathbf{u}+\mathbf{v}_{g}\right) \cdot \boldsymbol{\partial u}\right)+O\left(S t^{2}\right) .
$$

Expanding the exponential in (11) then provides the following expression for the drift $\boldsymbol{d}$ (with $\rho=\rho^{0}$ uniform)

$$
\boldsymbol{d}=\mathbf{v}_{g}-\int_{0}^{t}\left\langle\mathbf{u}(\mathbf{x}, t) \boldsymbol{\partial} \cdot \mathbf{V}\left(\boldsymbol{y}^{\prime}, t^{\prime}\right)\right\rangle d t^{\prime}+O\left(S t^{2}\right) .
$$

Reeks adopted a different approach, relating the velocity field to the Jacobian $J(t)=\left|\operatorname{det}\left[\boldsymbol{\partial} \boldsymbol{y}\left(t ; \mathbf{y}^{0}, 0\right) / \boldsymbol{\partial} \mathbf{y}^{0}\right]\right|$ such that $\dot{J}=(\boldsymbol{\partial} \cdot \mathbf{V}) J$, and treating $\varphi$ as a functional of the process $\mathbf{q}\left(t^{\prime}\right)=(\mathbf{V}, \boldsymbol{\partial} \cdot \mathbf{V})$. This permits a functional expansion for $\langle\varphi \mathbf{V}\rangle$ leading to the representation [19]

$$
\boldsymbol{d} \approx\langle\mathbf{V}\rangle-\int_{0}^{t}\left\langle\mathbf{V}^{*}(\mathbf{x}, t)(\boldsymbol{\partial} \cdot \mathbf{V})^{*}\left(\boldsymbol{y}^{\prime}, t^{\prime}\right)\right\rangle d t^{\prime}
$$

the superscript $*$ denoting zero-mean fluctuating variables.

A third formulation was developed by Chun et al. [20] with a particle velocity field defined in terms of conditionally averaged particle velocities; $\mathbf{V}(\mathbf{x}, t)=\langle\boldsymbol{v}(t)\rangle_{\mathbf{x}, \mathbf{u}}$, an 
ensemble, for a given realisation of $\mathbf{u}$, over initial states $\boldsymbol{z}^{0}$ such that $\boldsymbol{x}(t)=\mathbf{x}$. This leads to the representation

$$
\boldsymbol{d} \approx-\int_{0}^{t}\left\langle\mathbf{V}(\mathbf{x}, t) \boldsymbol{\partial} \cdot \mathbf{V}\left(\boldsymbol{y}^{\prime}, t^{\prime}\right)\right\rangle d t^{\prime}
$$

While there are clear similarities between the representations for $\boldsymbol{d}$ given by formulæ (13), (14) and (15) there are important differences: Maxey's model is, by construction, only appropriate in the small Stokes regime $(S t \ll 1)$. However, it does benefit from a particle velocity field that is defined a priori, by Eq. (12). Conversely, although the Reeks formulation given by Eq. (14) is valid for all $S t$ (and is exact if the underlying process $\mathbf{q}$ is Gaussian), the particle velocity field and its divergence must be inferred from the Jacobian determinant $J$. Moreover, the deformation tensor $\boldsymbol{\partial} \boldsymbol{y} / \boldsymbol{\partial} \mathbf{y}^{0}$ defining $J$ can exhibit temporal singularities. At such time-points $J=0$ and $\boldsymbol{\partial} \cdot \mathbf{V}$ is undefined (unbounded). This phenomenon, often interpreted in terms of caustics associated with crossing trajectories [6-8], poses a serious problem when attempting to evaluate the drift given by formula (14). The velocity field in the Chun et al. formulation is also problematic; it is precisely defined but the conditioned average is not a closed quantity. In principle this field and its divergence could be computed numerically from trajectory simulations, but in the absence of this it is difficult to evaluate the drift given by formula (15).

In contrast to all of the flux representations based on the notion of a particle velocity field, the formula given by Eq. (7) is based directly on the average fluid velocity sampled by particles, $\langle\varrho \mathbf{u}\rangle$. As such it perhaps provides a more useful starting point for attempting to construct closed-form expressions for the mass flux. We return to this aspect of the work in section IV. In preparation for that we first consider the role of symmetry reductions in helping to simplify the integrands in all the flux representations so far discussed.

\section{SYMMETRY REDUCTIONS}

All of the above models for the drift velocity $\boldsymbol{d}$ must respect basic symmetry properties inherent in the gravitational settling of particles in homogeneous flow. In particular, with co-ordinate axes $\mathrm{O} x_{1} x_{2} x_{3}$ such that $g_{i}=-g \delta_{1 i}$ it is evident that $d_{2}=d_{3}=0$. The aim here is to show that this condition is satisfied by each of the models defined by (7), (13), (14) and (15). In this respect the most challenging case is that of Eq. (7): From this definition of $\bar{\kappa}$ it is not obvious that $\bar{\kappa}_{2}=\bar{\kappa}_{3}=0$. Moreover, the representation of $\bar{\kappa}_{1}$ involves a summation of 9 terms. The symmetry analysis below addresses these points. The method also prompts reductions in closure expressions developed later, and translates naturally to analysis of other representations for $\boldsymbol{d}$.

To establish that $\bar{\kappa}_{2}, \bar{\kappa}_{3}$, as defined by Eq. (7), are identically zero we first note that, intuitively, the pdf $\phi(\mathbf{r})$ of $\boldsymbol{r}=\boldsymbol{x}\left(t^{\prime}\right)-\mathbf{x}$, satisfying $\boldsymbol{x}(t)=\mathbf{x}$, is an even function of $\left(\mathrm{r}_{2}, \mathrm{r}_{3}\right)$. That is $\phi\left(\mathrm{r}_{1},-\mathrm{r}_{2},-\mathrm{r}_{3}\right)=\phi\left(\mathrm{r}_{1}, \mathrm{r}_{2}, \mathrm{r}_{3}\right)$, and hence $\left\langle r_{2}\right\rangle=\left\langle r_{3}\right\rangle=0$. We wish to formalize and extend this to the distribution of $\boldsymbol{Z}\left(t^{\prime} ; \mathbf{x}, t\right)=(\boldsymbol{r}, \mathcal{H})$. Here we have the interpretation that $\mathcal{H}\left(t ; t^{\prime}\right)$, like $\boldsymbol{r}\left(t^{\prime}\right)$, is to be considered for realisations such that $\boldsymbol{x}(t)=\mathbf{x}$. From Eqs. (4), (5) and (8) $\boldsymbol{r}$ and $\mathcal{H}$ will satisfy

$$
\begin{aligned}
& \boldsymbol{r}\left(t^{\prime}\right)=h\left(t^{\prime}, t\right) \mathbf{v}-\frac{1}{\tau} \int_{t^{\prime}}^{t} h\left(t^{\prime}, s\right)\left(\boldsymbol{u}(s)+\mathbf{v}_{g}\right) d s \\
& \mathcal{H}\left(t ; t^{\prime}\right)=h\left(t, t^{\prime}\right) \mathrm{I}+\frac{1}{\tau} \int_{t^{\prime}}^{t} h(t, s) \boldsymbol{\Gamma}(s) \cdot \mathcal{H}\left(s ; t^{\prime}\right) d s,
\end{aligned}
$$

with $h(t, s)=\tau(1-\exp (-(t-s) / \tau)), \mathbf{v}=\dot{\boldsymbol{x}}(t)$, and $\boldsymbol{u}(s)=\mathbf{u}(\boldsymbol{r}(s)+\mathbf{x}, s)$. Now consider orthonormal co-ordinate transforms $\widetilde{\mathbf{x}}=\mathrm{P} \cdot \mathbf{x}$, such that the coordinate axis in the gravitational direction is unchanged, i.e. $\widetilde{\mathrm{x}}_{1}=\mathrm{x}_{1}$. Thus $\widetilde{\mathbf{v}}_{g}=\mathrm{P} \cdot \mathbf{v}_{g}=\mathbf{v}_{g}$. Define the process

$$
\widetilde{\boldsymbol{Z}}\left(t^{\prime} ; \widetilde{\mathbf{x}}, t\right)=(\widetilde{\boldsymbol{r}}, \widetilde{\mathcal{H}})=\left(\mathrm{P} \cdot \boldsymbol{r}, \mathrm{P} \cdot \mathcal{H} \cdot \mathrm{P}^{\top}\right)=\mathbb{P}(\boldsymbol{Z})
$$

Then $\widetilde{\boldsymbol{r}}, \widetilde{\mathcal{H}}$ will satisfy the transformed versions of (16), (17), involving $\widetilde{\mathbf{x}}=\mathrm{P} \cdot \mathbf{x}, \widetilde{\mathbf{v}}=\mathrm{P} \cdot \mathbf{v}, \widetilde{\mathbf{u}}(\widetilde{\mathbf{x}}, t)=\mathrm{P} \cdot \mathbf{u}(\mathbf{x}, t)$, and $\widetilde{\boldsymbol{\Gamma}}=\mathrm{P} \cdot \boldsymbol{\Gamma} \cdot \mathrm{P}^{\top}$. Since $\widetilde{\mathbf{v}}_{g}=\mathbf{v}_{g}$ it follows that $\widetilde{\boldsymbol{Z}}$ constitutes a valid realisation of the system associated with the corresponding realisation $\widetilde{\mathbf{u}}$. Moreover, since the averaged particle concentration field remains spatially uniform, the distribution of $\boldsymbol{r}$, and therefore of $\mathcal{H}$, will be independent of $\mathbf{x}$. It follows that the random variables $\widetilde{\boldsymbol{Z}}$ and $\boldsymbol{Z}$ have the same distribution, that is $\phi_{\widetilde{\boldsymbol{Z}}}(\boldsymbol{\zeta})=\phi_{\boldsymbol{Z}}(\boldsymbol{\zeta})$. From this it follows that, for any function $F(\boldsymbol{\zeta})$,

$$
\langle F(\boldsymbol{Z})\rangle=\langle F(\widetilde{\boldsymbol{Z}})\rangle=\langle F(\mathbb{P}(\boldsymbol{Z}))\rangle .
$$

To apply this to the integrand of $\bar{\kappa}$ in $(7)$ we shall need to consider sets of functions $F_{\mathbf{n}}(\boldsymbol{\zeta})$ indexed by $\mathbf{n} \in \mathrm{S}^{3}$, $\mathrm{S}=\{1,2,3\}$. There are two cases of interest. The first is when $F_{\mathbf{n}}(\mathbb{P}(\boldsymbol{\zeta}))=-F_{\mathbf{n}}(\boldsymbol{\zeta})$, in which case it follows that $\left\langle F_{\mathbf{n}}(\boldsymbol{Z})\right\rangle=-\left\langle F_{\mathbf{n}}(\boldsymbol{Z})\right\rangle=0$. The second case is $F_{\mathbf{n}}(\mathbb{P}(\boldsymbol{\zeta}))=F_{\mathbf{m}}(\boldsymbol{\zeta})$ giving $\left\langle F_{\mathbf{n}}(\boldsymbol{Z})\right\rangle=\left\langle F_{\mathbf{m}}(\boldsymbol{Z})\right\rangle$.

To establish simplifications for $\bar{\kappa}$ a number of symmetry transforms $\mathrm{P}$ need to be considered. Specifically, $\mathrm{P}_{1}=\operatorname{diag}(1,-1,-1), \mathrm{P}_{2}=\operatorname{diag}(1,-1,1)$ and

$$
\mathrm{P}_{3}=\left(\begin{array}{lll}
1 & 0 & 0 \\
0 & 0 & 1 \\
0 & 1 & 0
\end{array}\right), \quad \mathrm{P}_{4}=\left(\begin{array}{rrr}
1 & 0 & 0 \\
0 & 0 & -1 \\
0 & 1 & 0
\end{array}\right) \text {. }
$$

Table I gives the relationship between the components of $\widetilde{Z}$ and those of $\boldsymbol{Z}$ for each of these four transforms.

Now consider the integrand $\left\langle\mathcal{H}_{k j} \partial_{k} \mathrm{R}_{j i}\right\rangle_{\mathbf{x}}$ of $\bar{\kappa}_{i}$. Write

$$
\mathcal{H}_{k j}\left(t ; t^{\prime}\right) \partial_{k} \mathrm{R}_{j i}\left(\boldsymbol{r}, t^{\prime}-t\right)=\mathrm{F}_{k j i}\left(\boldsymbol{Z} ; t^{\prime}, t\right) .
$$

Then, in the transformed frame (omitting explicit reference to the time dependencies) $F_{k j i}(\widetilde{\boldsymbol{Z}})=\widetilde{\mathcal{H}}_{k j} \widetilde{\partial}_{k} \widetilde{\mathrm{R}}_{j i}$ 


\begin{tabular}{|c|c|c|c|c|}
\hline$m$ & $\widetilde{\boldsymbol{r}}$ & & $\widetilde{\mathcal{H}}$ & \\
\hline 1 & $\left(\begin{array}{l}+r_{1} \\
-r_{2} \\
-r_{3}\end{array}\right)$ & $\left(\begin{array}{l}+\mathcal{H}_{11} \\
-\mathcal{H}_{21} \\
-\mathcal{H}_{31}\end{array}\right.$ & $\begin{array}{l}-\mathcal{H}_{12} \\
+\mathcal{H}_{22} \\
+\mathcal{H}_{32}\end{array}$ & $\left.\begin{array}{l}-\mathcal{H}_{13} \\
+\mathcal{H}_{23} \\
+\mathcal{H}_{33}\end{array}\right)$ \\
\hline 2 & $\left(\begin{array}{l}+r_{1} \\
-r_{2} \\
+r_{3}\end{array}\right)$ & $\left(\begin{array}{l}+\mathcal{H}_{11} \\
-\mathcal{H}_{21} \\
+\mathcal{H}_{31}\end{array}\right.$ & $\begin{array}{l}-\mathcal{H}_{12} \\
+\mathcal{H}_{22} \\
-\mathcal{H}_{32}\end{array}$ & $\left.\begin{array}{l}+\mathcal{H}_{13} \\
-\mathcal{H}_{23} \\
+\mathcal{H}_{33}\end{array}\right)$ \\
\hline 3 & $\left(\begin{array}{l}+r_{1} \\
+r_{3} \\
+r_{2}\end{array}\right)$ & $\left(\begin{array}{l}+\mathcal{H}_{11} \\
+\mathcal{H}_{31} \\
+\mathcal{H}_{21}\end{array}\right.$ & $\begin{array}{l}+\mathcal{H}_{13} \\
+\mathcal{H}_{33} \\
+\mathcal{H}_{23}\end{array}$ & $\left.\begin{array}{l}+\mathcal{H}_{12} \\
+\mathcal{H}_{32} \\
+\mathcal{H}_{22}\end{array}\right)$ \\
\hline 4 & $\left(\begin{array}{l}+r_{1} \\
-r_{3} \\
+r_{2}\end{array}\right)$ & $\left(\begin{array}{l}+\mathcal{H}_{11} \\
-\mathcal{H}_{31} \\
+\mathcal{H}_{21}\end{array}\right.$ & $\begin{array}{l}-\mathcal{H}_{13} \\
+\mathcal{H}_{33} \\
-\mathcal{H}_{23}\end{array}$ & $\left.\begin{array}{l}+\mathcal{H}_{12} \\
-\mathcal{H}_{32} \\
+\mathcal{H}_{22}\end{array}\right)$ \\
\hline
\end{tabular}

TABLE I: Components of $\widetilde{\boldsymbol{Z}}$ in terms of $\boldsymbol{Z}$ for $\mathrm{P}_{m}$.

where $\widetilde{\boldsymbol{\partial}}=\mathrm{P} \cdot \boldsymbol{\partial}$, and the relationship between the components of $\widetilde{\mathbf{R}}=\mathrm{P} \cdot \mathbf{R} \cdot \mathrm{P}^{\top}$ and those of $\mathbf{R}$ follows the same pattern as $\widetilde{\mathcal{H}}, \mathcal{H}$ set out in TABLE I. With $\mathrm{P}=\mathrm{P}_{1}$, $\widetilde{\boldsymbol{\partial}}=\left(\partial_{1},-\partial_{2},-\partial_{3}\right)$ and, from TABLE I,

$$
\mathrm{F}_{222}(\widetilde{\boldsymbol{Z}})=\widetilde{\mathcal{H}}_{22} \widetilde{\partial}_{2} \widetilde{\mathrm{R}}_{22}=\mathcal{H}_{22}\left(-\partial_{2}\right) \mathrm{R}_{22}=-\mathrm{F}_{222}(\boldsymbol{Z}),
$$

indicating $\left\langle\mathcal{H}_{22} \partial_{2} \mathrm{R}_{22}\right\rangle=0$. Likewise, using both $\mathrm{P}_{1}$ and $\mathrm{P}_{2}$ it follows that $\left\langle\mathcal{H}_{k j} \partial_{k} \mathrm{R}_{j i}\right\rangle=0, i=2,3$, all $k$ and $j$, and hence, as required, $\bar{\kappa}_{2}=\bar{\kappa}_{3}=0$.

To simplify $\bar{\kappa}_{1}$ use $\mathrm{P}_{3}$ : Then $\widetilde{\boldsymbol{\partial}}=\left(\partial_{1}, \partial_{3}, \partial_{2}\right)$ and, for example,

$$
\mathrm{F}_{221}(\widetilde{\mathbf{Z}})=\widetilde{\mathcal{H}}_{22} \widetilde{\partial}_{2} \widetilde{\mathrm{R}}_{21}=\mathcal{H}_{33} \partial_{3} \mathrm{R}_{31}=\mathrm{F}_{331}(\mathbf{Z}) .
$$

Therefore $\left\langle\mathcal{H}_{22} \partial_{2} R_{21}\right\rangle=\left\langle\mathcal{H}_{33} \partial_{3} R_{31}\right\rangle$. Similarly

$$
\begin{aligned}
\left\langle\mathcal{H}_{12} \partial_{1} \mathrm{R}_{21}\right\rangle & =\left\langle\mathcal{H}_{13} \partial_{1} \mathrm{R}_{31}\right\rangle, \\
\left\langle\mathcal{H}_{21} \partial_{2} \mathrm{R}_{11}\right\rangle & =\left\langle\mathcal{H}_{31} \partial_{3} \mathrm{R}_{11}\right\rangle, \\
\left\langle\mathcal{H}_{23} \partial_{2} \mathrm{R}_{31}\right\rangle & =\left\langle\mathcal{H}_{32} \partial_{3} \mathrm{R}_{21}\right\rangle .
\end{aligned}
$$

These results are seen to reflect the invariance of the system with respect to the interchange of the co-ordinate axes $\mathrm{Ox}_{2} \leftrightarrow \mathrm{Ox}_{3}$. Using these results, and the incompressibility condition $\boldsymbol{\partial} \cdot \mathbf{R}=\mathbf{0}$, we obtain the following reduced expression for the integrand in (7)

$$
\left\langle\mathcal{H}_{k j} \partial_{k} \mathrm{R}_{j 1}\right\rangle=\left\langle\left(\mathcal{H}_{11}-\mathcal{H}_{22}\right) \partial_{1} \mathrm{R}_{11}\right\rangle+2\left(\left\langle\mathcal{H}_{12} \partial_{1} \mathrm{R}_{21}\right\rangle+\left\langle\mathcal{H}_{21} \partial_{2} \mathrm{R}_{11}\right\rangle+\left\langle\mathcal{H}_{23} \partial_{2} \mathrm{R}_{31}\right\rangle\right)
$$

The remaining, non-zero contributions in (18) still require closure of course. This is considered in the next section, where further symmetry-based reductions are introduced. Here we note that, based on the relationship between the components of $\boldsymbol{\Gamma}$, the fluid velocity gradient tensor sampled along particle trajectories, and those of $\widetilde{\boldsymbol{\Gamma}}=\mathrm{P} \cdot \boldsymbol{\Gamma} \cdot \mathrm{P}^{\top}$ we can deduce that all non-diagonal components of $\langle\boldsymbol{\Gamma}(t)\rangle$ are identically zero. Therefore, the fluid rotation rates experienced by particles all have zero mean values. That is $\langle\boldsymbol{\Omega}\rangle=\frac{1}{2}\left\langle\boldsymbol{\Gamma}-\boldsymbol{\Gamma}^{\top}\right\rangle=\mathbf{0}$. Again this is in accord with the expectation that particles experience no biasing in the $x_{2}$ and $x_{3}$ directions, and indicates that the non-zero contribution to $\langle\boldsymbol{\Gamma}\rangle$ is solely a consequence of the averaged straining $\langle\boldsymbol{\Sigma}\rangle=\frac{1}{2}\left\langle\boldsymbol{\Gamma}+\boldsymbol{\Gamma}^{\top}\right\rangle$ experienced by particles. This highlights that, regardless of the physical origin of the mechanism responsible for modifying the gravitational settling velocity, this effect is associated with the preferential sampling of $\boldsymbol{\Sigma}$ rather than $\boldsymbol{\Omega}$. Nonetheless, this observation is not central to the causal mechanisms which underlie the enhancement in settling velocity, and so is not considered further in the present work. Such symmetry-based deductions should also be applicable to configurations with a higher particle loading [21, 22] since effects such as three-way coupling, originating from particle motion, are manifest through wakes in the velocity field that serve to locally amplify strain rates in the flow.

\section{CLOSURE MODELS}

While the drift velocity representations provided by Eqs. (7), (13), (14) and (15) offer various insights into the origin and nature of enhanced settling, these expressions do not, as they stand, constitute closed-form models. The expressions can, in principle, be computed and assessed from particle simulation studies but, without closure of the underlying ensemble averages, they do not offer explicit formulæ for the drift.

With regard to closures for $\bar{\kappa}$, previous studies have invariably made use of Green's function approximations to the response tensor $\mathcal{H}$. The influence of $\boldsymbol{\Gamma}$, the sampled fluctuating fluid velocity gradient tensor, is neglected in (17), thus reducing $\mathcal{H}$ to a deterministic form, namely $\mathcal{H}_{k j}\left(t ; t^{\prime}\right)=h\left(t, t^{\prime}\right) \delta_{k j}$. This has proved successful in cases where $\langle\mathbf{u}\rangle \neq \mathbf{0}$, see for example [12, 23-25]. Here, however, in the zero-mean regime of homogeneous isotropic flow, this level of modelling fails. The integrand in (7) reduces to $h\left(t, t^{\prime}\right)\left\langle\boldsymbol{\partial} \cdot \mathbf{R}\left(\boldsymbol{r}, t^{\prime}-t\right)\right\rangle$, which is identically zero for incompressible flows. This essential dependence of $\mathcal{H}$ on $\boldsymbol{\partial u}$ again emphasises the crucial role of fluid straining and vorticity on generating enhanced gravitational settling, and demands more sophisticated approaches to closure. To this end we make further use of the correlation splitting technique: Given a random 
variable $\boldsymbol{X}$ and a deterministic function $G(\mathbf{x})$ then [13]

$$
\langle\boldsymbol{X} G(\boldsymbol{X})\rangle=\langle\langle\boldsymbol{X}\rangle\langle\langle G(\boldsymbol{X})\rangle+\langle\langle\boldsymbol{X} \boldsymbol{X}\rangle \cdot\langle\boldsymbol{\partial} G(\boldsymbol{X})\rangle+\cdots,
$$

where $\langle\langle\cdot\rangle$ denotes cumulants of $\boldsymbol{X}$. In particular $\langle\langle\boldsymbol{X}\rangle\rangle=\langle\boldsymbol{X}\rangle$, and $\langle\boldsymbol{X} \boldsymbol{X}\rangle\rangle=\left\langle\boldsymbol{X}^{\prime} \boldsymbol{X}^{\prime}\right\rangle, \boldsymbol{X}^{\prime}=\boldsymbol{X}-\langle\boldsymbol{X}\rangle$. Further, if $\boldsymbol{X}$ is Gaussian then all higher-order cumulants are zero. Applying (19) to the integrand of $\bar{\kappa}_{1}$ gives (the conditionality $\boldsymbol{x}(t)=\mathbf{x}$ in the ensembles being implicit)

$$
\left\langle\mathcal{H}_{k j} \partial_{k} \mathrm{R}_{j 1}\right\rangle=\sum_{n=1}^{\infty} T_{n}
$$

in which the first 4 terms are given by the expressions

$$
\begin{aligned}
& T_{1}=\left\langle\left\langle\mathcal{H}_{k j}\right\rangle\right\rangle\left\langle\partial_{k} \mathrm{R}_{j 1}\right\rangle \\
& T_{2}=-\left\langle\left\langle\mathcal{H}_{k j} r_{n}\right\rangle\right\rangle\left\langle\partial_{n} \partial_{k} \mathrm{R}_{j 1}\right\rangle \\
& T_{3}=\left\langle\left\langle\mathcal{H}_{k j} r_{n} r_{p}\right\rangle\right\rangle\left\langle\partial_{p} \partial_{n} \partial_{k} \mathrm{R}_{j 1}\right\rangle \\
& T_{4}=-\left\langle\left\langle\mathcal{H}_{k j} r_{n} r_{p} r_{q}\right\rangle\right\rangle\left\langle\partial_{q} \partial_{p} \partial_{n} \partial_{k} \mathrm{R}_{j 1}\right\rangle
\end{aligned}
$$

We can again make use of symmetries to simplify the summations in each term of this expansion. Firstly, since $\phi_{\widetilde{\mathcal{H}}}=\phi_{\mathcal{H}}$ it follows from TABLE I that, for all $k \neq j$, $\mathcal{H}_{k j}$ has an even distribution and therefore zero-mean. Further, $\mathcal{H}_{22}$ and $\mathcal{H}_{33}$ have the same distribution. These, and the incompressibility condition $\boldsymbol{\partial} \cdot \mathbf{R}=\mathbf{0}$, reduce the first term $T_{1}$ to

$$
T_{1}=\left\langle\mathcal{H}_{11}-\mathcal{H}_{22}\right\rangle\left\langle\partial_{1} \mathrm{R}_{11}\right\rangle .
$$

To establish reductions in the second term $T_{2}$ define $F_{n k j}(\boldsymbol{r})=\partial_{n} \partial_{k} \mathrm{R}_{j 1}\left(\boldsymbol{r}, t^{\prime}-t\right)$. Then, with $\mathrm{P}=\mathrm{P}_{1}$,

$$
F_{222}(\widetilde{\boldsymbol{Z}})=\widetilde{\partial}_{2} \widetilde{\partial}_{2} \widetilde{\mathrm{R}}_{21}=\left(-\partial_{2}\right)\left(-\partial_{2}\right)\left(-\mathrm{R}_{21}\right)=-F_{222}(\boldsymbol{Z})
$$

implying that $\left\langle\partial_{2} \partial_{2} R_{21}\right\rangle=0$. Similar consideration of all the $F_{n k j}$ under $\mathrm{P}_{1}$ and $\mathrm{P}_{2}$ establishes that the only non-zero components of $\left\langle\partial_{n} \partial_{k} \mathrm{R}_{j 1}\right\rangle$ are

$$
\left\{\begin{array}{l}
\left\langle\partial_{1} \partial_{1} R_{11}\right\rangle \\
\partial_{2} \partial_{2} R_{11} \\
\partial_{3} \partial_{3} R_{11}
\end{array}\right\} \quad\left\{\begin{array}{l}
\left.\partial_{2} \partial_{1} R_{21}\right\rangle=\left\langle\partial_{1} \partial_{2} R_{21}\right\rangle \\
\left.\partial_{3} \partial_{1} R_{31}\right\rangle=\left\langle\partial_{1} \partial_{3} R_{31}\right.
\end{array}\right\rangle
$$

Transform $\mathrm{P}_{4}$ establishes $\left\langle\partial_{2} \partial_{2} \mathrm{R}_{11}\right\rangle=\left\langle\partial_{3} \partial_{3} \mathrm{R}_{11}\right\rangle$, and $\left\langle\partial_{2} \partial_{1} R_{21}\right\rangle=\left\langle\partial_{3} \partial_{1} R_{31}\right\rangle$. Combining these, and using $\boldsymbol{\partial} \cdot \mathbf{R}=\mathbf{0}$, reduces the 27 terms in $T_{2}$ to

$$
\begin{aligned}
T_{2} & =2\left\langle\left\langle\mathcal{H}_{21} r_{2}\right\rangle\right\rangle\left\langle\partial_{2} \partial_{2} \mathrm{R}_{11}\right\rangle \\
& +\left(\left\langle\left\langle\left(\mathcal{H}_{11}-\mathcal{H}_{22}\right) r_{1}\right\rangle-\left\langle\left\langle\mathcal{H}_{12} r_{2}\right\rangle\right)\left\langle\partial_{1} \partial_{1} \mathrm{R}_{11}\right\rangle .\right.\right.
\end{aligned}
$$

The cumulant expansion given by Eq. (20), when introduced into the flux representation given by Eq. (7) offers both a mechanism to formulate closures for this enhanced flux, as well as a means to identify the dominant contributions to this. These two avenues of investigation form the cornerstone of this work, and the assessment of each against numerical data is covered in sections VB and
V C. The expansion given by Eq. (20) reduces the closure problem to that of finding a strategy for constructing models for the cumulant correlations, $\mathbf{K}^{1}=\langle\langle\mathcal{H}\rangle\rangle$, $\mathbf{K}^{2}=\langle\langle\mathcal{H} \boldsymbol{r}\rangle\rangle, \mathbf{K}^{3}=\langle\langle\mathcal{H} \boldsymbol{r} \boldsymbol{r}\rangle\rangle$ etc., and for the averages of gradients in the fluid velocity correlation tensor $\mathbf{R}$ sampled along trajectories. To these ends we make use of the representations of $\boldsymbol{r}$ and $\mathcal{H}$ given by Eqs. (16), (17). Eq. (16) can be rewritten as

$\boldsymbol{r}\left(t^{\prime}\right)=\left(t^{\prime}-t\right) \mathbf{v}_{g}+h\left(t^{\prime}, t\right)\left(\mathbf{v}-\mathbf{v}_{g}\right)-\frac{1}{\tau} \int_{t^{\prime}}^{t} h\left(t^{\prime}, s\right) \boldsymbol{u}(s) d s$,

whilst, substituting representation Eq. (17) for $\mathcal{H}$ recursively into the right-hand side of the same equation leads to the approximation

$$
\mathcal{H}\left(t ; t^{\prime}\right) \approx h\left(t, t^{\prime}\right) \mathrm{I}+\frac{1}{\tau} \int_{t^{\prime}}^{t} h(t, s) h\left(s, t^{\prime}\right) \boldsymbol{\Gamma}(s) d s .
$$

Eq. (25) is a leading-order approximation, which is found to capture virtually all of the particle response behaviour which is important in the context of gravitational settling. The effect of including more detail from Eq. (17) in an explicit description of $\mathcal{H}$ is negligible, and higherorder contributions from Eq. (17) have therefore been omitted in the present work.

Eq. (24) is a starting point for formulating closures to the means of sampled gradients in $\mathbf{R}$ : Averaging over trajectories such that $\boldsymbol{x}(t)=\mathbf{x}$, and noting that, since the system under consideration is statistically homogeneous and stationary, $\langle\boldsymbol{u}(s)\rangle_{\mathbf{x}}=\overline{\boldsymbol{\kappa}}=\langle\mathbf{v}\rangle-\mathbf{v}_{g}$, gives the exact relationship $\left\langle\boldsymbol{r}\left(t^{\prime}\right)\right\rangle=\left(t^{\prime}-t\right)\langle\mathbf{v}\rangle$. This prompts the simple approximation $\langle\boldsymbol{r}\rangle \approx \boldsymbol{r}^{0}=\left(t^{\prime}-t\right) \mathbf{v}_{g}$, and then the closure

$$
\left\langle\partial_{k} \mathrm{R}_{j 1}\left(\boldsymbol{r}\left(t^{\prime}\right), t^{\prime}-t\right)\right\rangle \approx \partial_{k} \mathrm{Q}_{j 1}\left(\boldsymbol{r}^{0}\right) \mathrm{E}\left(t^{\prime}-t ; \tau_{L p}\right)
$$

where $\mathbf{Q}(\mathbf{r})=\mathbf{R}(\mathbf{r}, 0)$, and $\mathrm{E}\left(s ; \tau_{L p}\right)$ is a modified temporal decorrelation function that accounts for the influence of particle sampling by replacing the fluid Eulerian integral timescale, $\tau_{E}$, with a Lagrangian timescale, $\tau_{L p}$. A specific model for $\tau_{L p}$ is given in the Section $\mathrm{V}$ where results show that this closure strategy is very effective, not only for $\langle\boldsymbol{\partial} \mathbf{R}\rangle$ but also for higher-order derivatives.

With regard to forming closures for the correlations between $\mathcal{H}$ and $\boldsymbol{r}$ embedded in the cumulants $\mathbf{K}^{n}$, we note that Eqs. (24), (25) provide expressions for $\boldsymbol{r}$ and $\mathcal{H}$ in terms of, respectively, sampled fluid velocities $\boldsymbol{u}$ and fluid velocity gradients $\boldsymbol{\Gamma}$. In consequence the cumulants $\mathbf{K}^{n}$ can be reduced to correlations between $\boldsymbol{u}$ and $\boldsymbol{\Gamma}$, and these are amenable to further closure modelling.

Firstly, averaging Eq. (25) introduces the unclosed term $\langle\boldsymbol{\Gamma}(s)\rangle_{\mathbf{x}}$ into the approximation for $\langle\langle\mathcal{H}\rangle\rangle$. A representation for this unclosed term can be derived using the same approach used to represent $\langle\mathbf{u}\rangle_{\mathbf{x}}$ in terms of $\overline{\boldsymbol{\kappa}}$. 
This gives (see Appendix C)

$$
\left\langle\Gamma_{i m}(t)\right\rangle_{\mathbf{x}} \approx \int_{0}^{t}\left\langle\mathcal{H}_{k j}\left(t ; t^{\prime}\right) \partial_{m} \partial_{k} \mathrm{R}_{j i}\left(\boldsymbol{r}, t^{\prime}-t\right)\right\rangle_{\mathbf{x}} d t^{\prime}
$$

We note that the integrand in Eq. (27) is zero if $m \neq i$, indicating that this approximation respects the symmetry constraint that requires $\langle\boldsymbol{\Gamma}\rangle$ and $\langle\mathcal{H}\rangle$ to be diagonal.
A cumulant expansion in Eq. (27) gives

$$
\begin{aligned}
\left\langle\mathcal{H}_{k j} \partial_{m} \partial_{k} \mathrm{R}_{j i}\right\rangle & =\left\langle\left\langle\mathcal{H}_{k j}\right\rangle\right\rangle\left\langle\partial_{m} \partial_{k} \mathrm{R}_{j i}\right\rangle \\
& +\left\langle\langle \mathcal { H } _ { k j } r _ { n } \rangle \left\langle\left\langle\partial_{n} \partial_{m} \partial_{k} \mathrm{R}_{j i}\right\rangle+\cdots\right.\right.
\end{aligned}
$$

Symmetry considerations reduce the first term in this expansion to

$$
\left\langle\left\langle\mathcal{H}_{k j}\right\rangle\right\rangle\left\langle\partial_{m} \partial_{k} \mathrm{R}_{j i}\right\rangle=\delta_{i m}\left\langle\mathcal{H}_{11}-\mathcal{H}_{22}\right\rangle\left\langle\partial_{m} \partial_{1} \mathrm{R}_{1 i}\right\rangle .
$$

Of course this introduces the mean value $\langle\mathcal{H}\rangle$ again. However, results show that this contribution is small compared to the second term in Eq. (28). Indeed, the dominant contributions in both Eqs. (20) and (28) are those associated with the second-order cumulants. These can be approximated by combining Eqs. (24) and (25) to get

$$
\left\langle\left\langle\mathcal{H}_{k j} r_{n}\right\rangle \approx \frac{1}{\tau} h\left(t^{\prime}, t\right) \int_{t^{\prime}}^{t} h(t, s) h\left(s, t^{\prime}\right)\left\langle\Gamma_{k j}^{\prime}(s) \mathrm{v}_{n}^{\prime}\right\rangle d s-\frac{1}{\tau^{2}} \int_{t^{\prime}}^{t} h\left(t, s_{1}\right) h\left(s_{1}, t^{\prime}\right) \int_{t^{\prime}}^{t} h\left(t^{\prime}, s_{2}\right)\left\langle\Gamma_{k j}^{\prime}\left(s_{1}\right) u_{n}^{\prime}\left(s_{2}\right)\right\rangle d s_{2} d s_{1}\right.
$$

where $\mathbf{v}^{\prime}, \boldsymbol{u}^{\prime}$ and $\boldsymbol{\Gamma}^{\prime}$ denote fluctuations about mean values. A natural closure for $\left\langle\boldsymbol{\Gamma}^{\prime}\left(s_{1}\right) \boldsymbol{u}^{\prime}\left(s_{2}\right)\right\rangle$ is obtained by taking $\left\langle\boldsymbol{\Gamma}\left(s_{1}\right)\right\rangle\left\langle\boldsymbol{u}\left(s_{2}\right)\right\rangle \approx 0$ and then

$$
\begin{aligned}
\left\langle\Gamma_{k j}^{\prime} u_{n}^{\prime}\right\rangle & \approx\left\langle\partial_{j} \mathrm{u}_{k}\left(\boldsymbol{x}\left(s_{1}\right), s_{1}\right) \mathrm{u}_{n}\left(\boldsymbol{x}\left(s_{2}\right), s_{2}\right)\right\rangle \\
& \approx\left\langle\partial_{j} \mathrm{R}_{k n}\left(\boldsymbol{r}\left(s_{1}\right)-\boldsymbol{r}\left(s_{2}\right), s_{1}-s_{2}\right)\right\rangle .
\end{aligned}
$$

The closure strategy given by Eq. (26) can then be used. For the correlation $\left\langle\boldsymbol{\Gamma}^{\prime}(s) \mathbf{v}^{\prime}\right\rangle$, we relate particle velocities to sampled fluid velocities via a particle-fluid stochastic model [26], further details of which are outlined in Appendix D. This provides expressions for correlations between these velocities, and to conform with these we take

$$
\mathbf{v} \approx u^{\prime} \frac{\sqrt{S t_{E}}}{1+S t_{E}} \boldsymbol{z}+\frac{1}{1+S t_{E}} \boldsymbol{u}\left(t^{\prime}\right)+\mathbf{v}_{g} .
$$

Here $u^{\prime}$ is the root-mean-square velocity for the fluid, $S t_{E}=\tau / \tau_{E}$, and $\boldsymbol{z}$ is the standard normal deviate. The correlation $\left\langle\boldsymbol{\Gamma}^{\prime}(s) \mathbf{v}^{\prime}\right\rangle$ is therefore modelled as

$$
\left\langle\boldsymbol{\Gamma}^{\prime}(s) \mathbf{v}^{\prime}\right\rangle \approx \frac{1}{1+S t_{E}}\left\langle\boldsymbol{\Gamma}^{\prime}(s) \boldsymbol{u}^{\prime}\left(t^{\prime}\right)\right\rangle,
$$

and Eq. (31) can then be used again. Thus the same strategy is used to model both $\left\langle\boldsymbol{\Gamma}^{\prime}\left(s_{1}\right) \boldsymbol{u}^{\prime}\left(s_{2}\right)\right\rangle$ and $\left\langle\boldsymbol{\Gamma}^{\prime}(s) \mathbf{v}^{\prime}\right\rangle$.

The effectiveness of these closures is discussed in the next section. A similar approach could be considered for terms $T_{3}$ and $T_{4}$ in expansion (20). This is not pursued here since the results in Section $V$ demonstrate that, in the present context, the contribution of these terms to the enhanced settling is secondary.

\section{RESULTS AND DISCUSSION}

Various representations for the particle mass flux $\rho \overline{\mathbf{v}}$ were set out in section II. These all purport to capture (subject to associated provisos) the increase in settling velocity experienced by inertial particles in a gravitational field. However, that this is the case is not immediately obvious, and therefore it is instructive to assess such models making use of particle-trajectory simulations. Results from such simulations also provide a benchmark against which the closure modelling strategy developed in section IV can be assessed. As noted in section II, two of the formulations, Eqs. (14) and (15), are problematic in that they do not lend themselves easily to a simulation study. We therefore confine our attention to the flux representations given by Eqs. (7) and (13).

In the case of the representation given by Eq. (7), the claim is that this provides an exact description of enhanced settling subject only to the underlying fluid velocity field $\mathbf{u}$ conforming to a Gaussian distribution. With this in mind we have adopted a kinematic simulation (KS) approach for generating stochastic fields. This allows for the construction of velocity fields that are not only strictly Gaussian, but that also reproduce prescribed forms for fluid velocity correlations and turbulent kinetic energy spectra consistent with the general theory of homogeneous, isotropic turbulence. In this way, with a closed form expression for $\mathbf{R}$, we ensure that no confounding factors have to be taken into account when assessing our closure modelling. We have chosen to simulate the velocity field $\mathbf{u}$ such that this reproduces the Batchelor-Townsend energy spectrum. Details of the 
simulation method are given in Appendix A.

By computing particle trajectories in these simulated flows we can investigate the validity and accuracy of the flux representations. Further, in the case of that given by Eq. (7), we can assess both the relative contributions to this flux from the terms $T_{n}$ in the expansion (20), and the performance of the proposed closure models for these terms. These investigations are presented next.

\section{A. Assessment of Flux Representations}

The representations for enhanced settling given by Eqs. (7) and (13) can be assessed from two measures obtainable from simulation data: Firstly, by direct evaluation of the mean particle velocity $\overline{\mathbf{v}}$. This, of course, provides the reference value, $\overline{\mathbf{v}}-\mathbf{v}_{g}$. Alternatively, from Eq. (6), the preferentially sampled fluid velocity $\langle\varrho \mathbf{u}\rangle$ should be equivalent.

Simulations were performed with a range of parameter values, $0.05<S t_{E}<5$ and $0.05<V_{g}=\left|\mathbf{v}_{g}\right|<2$, in a two-dimensional KS flow field. All simulations used $10^{6}$ particles and were run in parallel on 44 processors for a period of $3 \tau+3 \tau_{E}$ to achieve equilibrated statistical measures, and a further $4 \tau_{L p}$ for the sampling of statistics. Fig. 1 shows corresponding results for the 3 measures of enhanced settling; $\overline{\mathbf{v}}-\mathbf{v}_{g},\langle\varrho \mathbf{u}\rangle$, and $\overline{\boldsymbol{\kappa}}$. The last of these is computed using the simulated particle trajectories to perform a direct numerical evaluation of the expression given by Eq. (7). All results are normalised with respect to the fluid root mean square velocity $u^{\prime}$, and error bars represent the standard deviation from time-averaging. From the figure it is seen that the modification in settling velocity is always positive, which is consistent with the use of the linear drag model (5) [27, 28], and furthermore the peak increase of around $7 \%$ is in line with previous findings using synthetic velocity fields [1].

The results are seen to substantiate the validity of the enhanced flux representation provided by $\bar{\kappa}$ across the full range of values of $S t_{E}$ and $V_{g}$. This demonstrates that, for a Gaussian flow field, the functional correlation splitting approach in (7) is able to capture all the physical mechanisms which act to cause the increase in settling velocity that is observed, and is therefore a suitable framework for model development of this phenomenon. The capacity of this formulation to capture the enhanced settling stems from the interaction between $\mathcal{H}$ and $\boldsymbol{\partial R}$ as seen by particles that is intrinsic to Eq. (7). This implies that it is the correlation between the path history of particles and the sampling of spatio-temporal flow structures along trajectories which is responsible for the increase in settling velocity, and this is in accordance with the preferential concentration phenomenology [1, 29].

It is worth noting that in principle the functional correlation splitting approach can be extended to include higher-order cumulants which account for the nonGaussian aspects of the flow [15], meaning that this approach is capable of capturing the range of various phys-

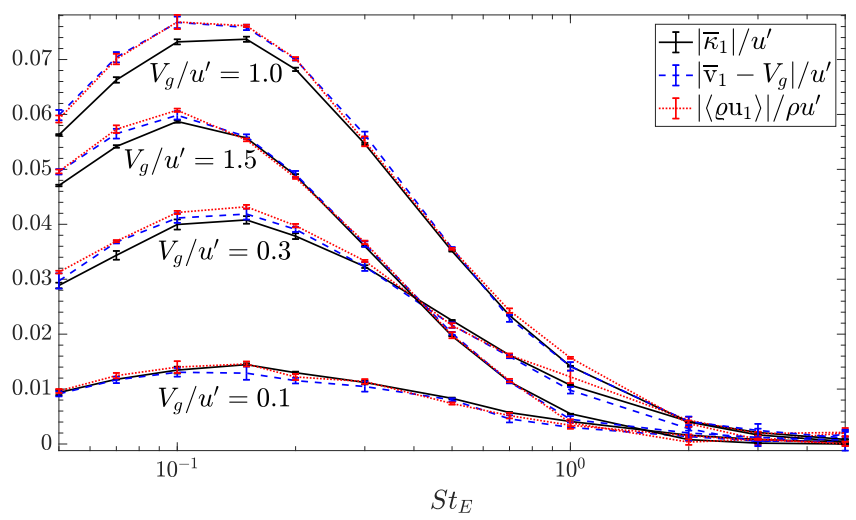

FIG. 1: (Color online) Enhanced settling velocity. Comparison of $\left|\bar{\kappa}_{1}\right| / u^{\prime}$ with $\left|\overline{\mathrm{v}}_{1}-V_{g}\right| / u^{\prime}$ and $\left|\left\langle\varrho u_{1}\right\rangle\right| / \rho u^{\prime}$.

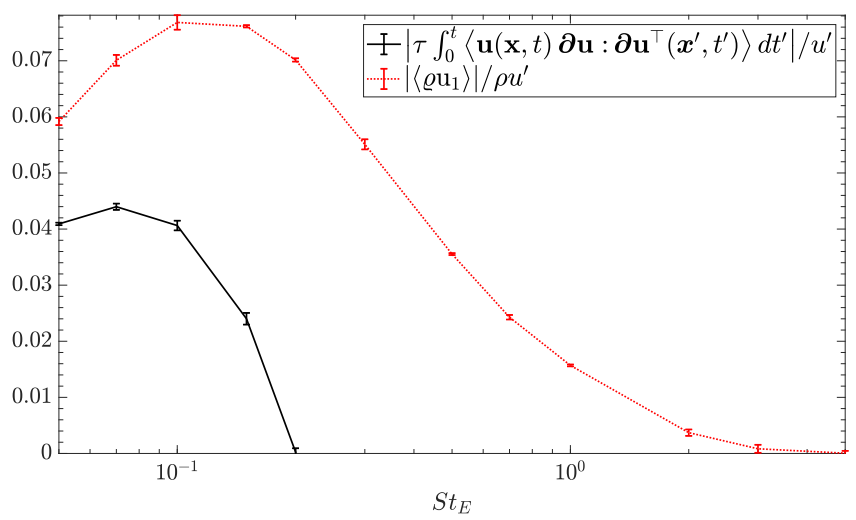

FIG. 2: (Color online) Enhanced settling velocity. Comparison of (34) with $\left|\left\langle\varrho u_{1}\right\rangle\right| / \rho u^{\prime}, V_{g} / u^{\prime}=1.0$.

ical mechanisms observed for particles settling in true turbulence [21, 22, 28]. The additional contributions are generalisations of the history integral given by $\bar{\kappa}$ in Eq. (7) and are infinite in number, however the expression for $\overline{\boldsymbol{\kappa}}$ remains the leading order contribution. Consequently $\bar{\kappa}$ would still be expected to capture the dominant contribution to the drift velocity in true turbulence across a range of Reynolds numbers. The methodology can also be extended to non-linear drag models for describing the particle trajectories, in which case the functional correlation splitting approach would be expected to account for the phenomenon of loitering observed at large $S t[27,28]$.

Fig. 2 shows a similar comparison between the reference values $\langle\varrho \mathbf{u}\rangle$ and the representation given by equation (13), using expression (12) for $\mathbf{V}$ to reduce this to the approximation

$$
\langle\varrho \mathbf{u}\rangle \approx \tau \rho \int_{0}^{t}\left\langle\mathbf{u}(\mathbf{x}, t) \boldsymbol{\partial u}: \boldsymbol{\partial} \mathbf{u}^{\top}\left(\boldsymbol{x}^{\prime}, t^{\prime}\right)\right\rangle d t^{\prime} .
$$

The results shown were obtained using a normalised gravitational settling velocity $V_{g} / u^{\prime}=1$ which, from 
Fig. 1, maximises the enhanced setting. As can be seen, the agreement provided by approximation (34) is not strong. Of course, by construction, we would not expect good agreement for $S t \sim \mathcal{O}(1)$. However, even for $S t \sim \mathcal{O}(0.1)$ the agreement is limited, and only the underlying, qualitative trend is recovered. This demonstrates that the centrifuge process identified by the small $S t$ analysis is not the only mechanism responsible for enhanced settling, with other mechanisms predominant at larger St. This is the case even in the simplified context of a Gaussian flow field, and highlights the importance of the multiscale effects that underlie this phenomenon [29].

The contrast with Fig. 1 is striking, and prompts further assessment of the preceding analysis aimed at constructing closure models for the flux representation provided by Eq. (7). Results from these investigations are presented next.

\section{B. Relative Contributions of Cumulants}

In terms of modelling, it is instructive to first numerically evaluate the terms in the cumulant expansion (20) for the integrand of $\bar{\kappa}_{1}$, in order to ascertain the importance of their relative contributions. Although the flow field $\mathbf{u}$ is Gaussian, the resulting processes $\boldsymbol{r}$ and $\mathcal{H}$ are not, and this necessitates the investigation of at least some higher-order terms. We consider the first four terms, $T_{1}$ to $T_{4}$. These have been evaluated using simulated particle trajectories, and illustrative results are shown in Fig. 3. These results were obtained using $S t_{E}=0.1$ and $V_{g} / u^{\prime}=1.0$, corresponding to the maximum enhanced settling (Fig. 1). Fig. 3 shows the (normalised) time evolution of the integrand $\left\langle\mathcal{H}_{k j} \partial_{k} \mathrm{R}_{j 1}\right\rangle$ together with the contributions to this of the different expansion terms $T_{n}$. It is immediately apparent that the dominant contribution arises from $T_{2}$, and is thus associated with the second cumulant $\mathbf{K}^{2}=\langle\langle\mathcal{H} \boldsymbol{r}\rangle\rangle$. The contributions from the other terms, $T_{1}, T_{3}$, and $T_{4}$, are all a full order of magnitude smaller, and consequently play a substantially less significant role in determining the overall particle settling velocity. Nonetheless, the presence of non-zero $T_{3}$ and $T_{4}$ highlights non-Gaussian aspects in the joint distribution of $\boldsymbol{r}$ and $\mathcal{H}$ which arise from the biasing that is inherent in particle trajectories.

Another way to illustrate the relative importance of terms $T_{1}$ through $T_{4}$ is by considering the cumulative contribution of these to the overall behaviour of the integrand $\left\langle\mathcal{H}_{k j} \partial_{k} \mathrm{R}_{j 1}\right\rangle$. This is illustrated in Fig. 4. Clearly $T_{1}$ cannot, by itself, be considered a meaningful descriptor of this average. However, the partial sum $T_{1}+T_{2}$ is seen to account for the majority of the average, due to the dominant contribution of $T_{2}$. The addition of $T_{3}$ provides only marginally greater accuracy, whilst inclusion of $T_{4}$ adds a slightly more substantial contribution. At this level of description, the remaining deficiency is concentrated around the peak amplitude of the average $\left\langle\mathcal{H}_{k j} \partial_{k} \mathrm{R}_{j 1}\right\rangle$, and also at large time separations of $\left(t-t^{\prime}\right)$.

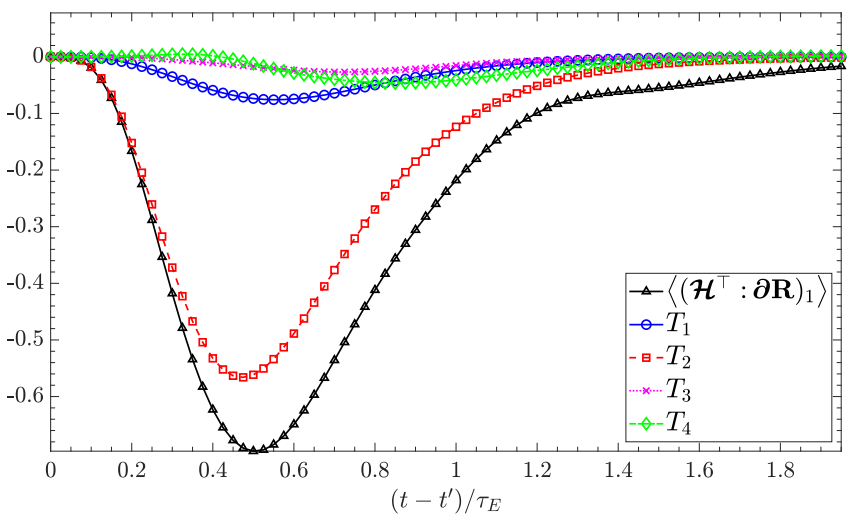

FIG. 3: (Color online) Contribution of terms $T_{n}$ to the average $\left\langle\left(\mathcal{H}^{\top}: \boldsymbol{\partial R}\right)_{1}\right\rangle, S t_{E}=0.1, V_{g} / u^{\prime}=1.0$.

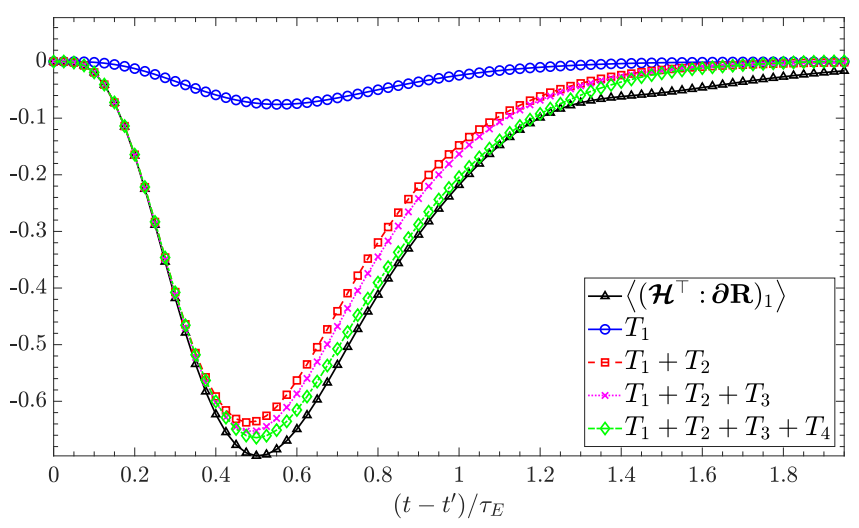

FIG. 4: (Color online) Partial sums of $T_{n}$ as approximations to $\left\langle\left(\mathcal{H}^{\top}: \partial \mathbf{R}\right)_{1}\right\rangle, S t_{E}=0.1, V_{g} / u^{\prime}=1.0$.

This implies that contributions from additional terms, $T_{5}, T_{6}$ etc., in the expansion will make little discernible difference, and justifies truncation of the expansion at $T_{4}$. Furthermore, it is seen in Fig. 4 that the information contained within $T_{1}$ and $T_{2}$ constitutes the majority of the variation in $\left\langle\mathcal{H}_{k j} \partial_{k} \mathrm{R}_{j 1}\right\rangle$, and is certainly sufficient to capture the characteristic behaviour that is responsible for the increase in particle settling velocity.

The relatively small contribution from $T_{1}$ can be explained by recalling that, as deduced based on symmetry arguments in section III, $\mathbf{K}^{1}=\langle\langle\mathcal{H}\rangle\rangle$ is diagonal. The contraction with $\left\langle\boldsymbol{\partial R}\left(\boldsymbol{r}, t^{\prime}-t\right)\right\rangle$ then results in terms that approximately cancel, in accord with the incompressibility condition $\boldsymbol{\partial} \cdot \mathbf{R}=\mathbf{0}$. In contrast, $\mathbf{K}^{2}=\langle\langle\mathcal{H} \boldsymbol{r}\rangle\rangle$ in $T_{2}$ reflects to the interaction between $\boldsymbol{r}$ and $\mathcal{H}$ which is associated with the non-local path history effects that are sampled along individual trajectories $[2,5]$, and only this level of detail is sufficient to account for the majority of the increase in particle settling velocity. This emphasises that the mechanisms which underlie this phenomenon are not directly dependent on average properties of either the flow field or the particle phase, but rather the collective influence of the flow field on individual particles, and 
specifically how the trajectory dependent response tensor $\mathcal{H}$ and separation along trajectories $\boldsymbol{r}$ interact with each other. This also explains why Green's function approximations to the response tensor $\mathcal{H}$ are incapable of capturing any of the settling velocity enhancement experienced by particles; these approximations effectively model the average particle response $\langle\mathcal{H}\rangle$ by omitting the individual trajectory history dependence of particles, and therefore only have the capacity to include information contained within $T_{1}$. This highlights the necessity to account for trajectory dependent history effects and, in particular, the key role of the fluid velocity gradient tensor sampled by particles. On this basis a carefully constructed closure model for $T_{2}$ will be sufficient to capture essential features modifying the particle settling velocity. This is in contrast to previous modelling work using the functional correlation splitting approach in cases where $\langle\mathbf{u}\rangle \neq \mathbf{0}$ $[12,23-25]$. In these scenarios the average particle phase behaviour is more important than the individual trajectory dependence on the flow field. Consequently, in such flows, $T_{1}$ rather than $T_{2}$ gives the dominant contribution to the mass flux.

\section{Closure Model Assessment}

The closure strategy outlined in section IV was applied to the first two terms, $T_{1}$ and $T_{2}$, of the expansion (20). The application to terms $T_{3}$ and $T_{4}$ was not considered; partly because of the added complexity involved, but also because, as we have just seen, these terms contribute a relatively minor component to the overall mass flux. In this section therefore attention is confined to assessing the approach as applied to $T_{1}$ and $T_{2}$.

The model requires specification of a suitable Lagrangian decorrelation function in Eq. (26). We take $\mathrm{E}\left(s ; \tau_{L p}\right)=\exp \left[-\frac{\pi}{4 \tau_{L p}^{2}} s^{2}\right]$. This is a natural variation of the decorrelation function defined in Eq. (A2), with a modification that seeks to accounts for the influence of particle trajectories by replacing the fluid Eulerian integral timescale $\tau_{E}$ with $\tau_{L p}$. The specific model used for $\tau_{L p}$ is the empirical relation [30]

$$
\tau_{L p}=\tau_{E}-\frac{\tau_{E}-\tau_{L}}{\left(1+S t_{E}\right)^{-0.4\left(1+0.01 S t_{E}\right)}},
$$

where $\tau_{L}$ is the Lagrangian fluid integral timescale as determined from the specific flow field under consideration. Use of Eq. (35) ensures that model (31) is physically consistent with the correlation being that of fluid quantities sampled by particles, and that the associated decorrelation timescale is then representative of particle settling.

The ability of the proposed closure strategy to accurately capture the increase in settling velocity depends upon the effectiveness of the models for the various expressions in the expansion (20); specifically the spatial derivatives of $\mathbf{R}$ evaluated along particle trajectories as modelled in Eq. (26), and the cumulants $\langle\langle\mathcal{H}\rangle\rangle$ and $\langle\langle\mathcal{H} \boldsymbol{r}\rangle\rangle$

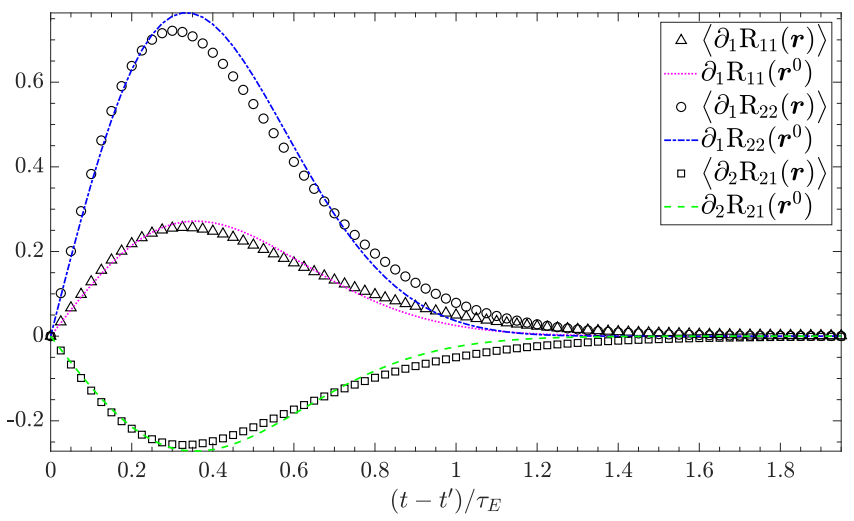

FIG. 5: (Color online) Evaluation of closure approximations $\langle\partial \mathbf{R}(\boldsymbol{r})\rangle \approx \partial \mathbf{R}\left(\boldsymbol{r}^{0}\right), S t_{E}=0.1, V_{g} / u^{\prime}=1.0$.

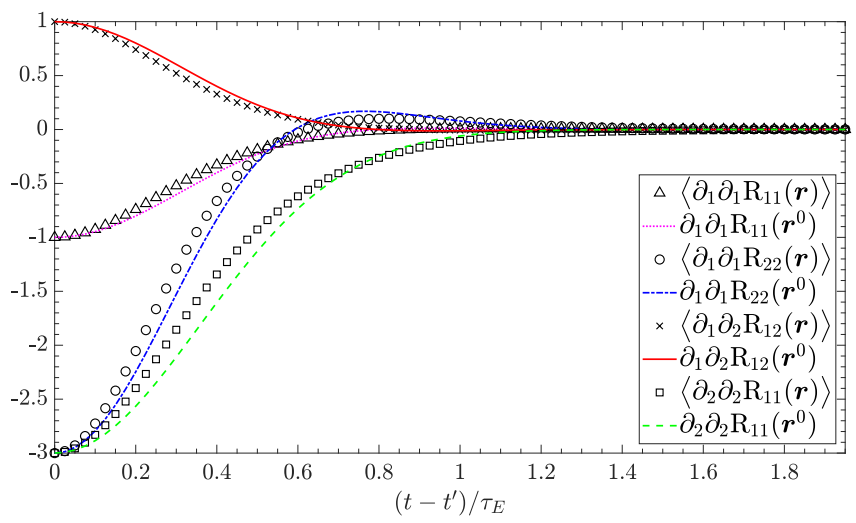

FIG. 6: (Color online) Evaluation of closure approximations $\langle\boldsymbol{\partial} \boldsymbol{\partial R}(\boldsymbol{r})\rangle \approx \boldsymbol{\partial} \boldsymbol{\partial R}\left(\boldsymbol{r}^{0}\right), S t_{E}=0.1, V_{g} / u^{\prime}=1.0$.

themselves, which are modelled using Eqs. (25), (27) and (30). The former closure completely neglects the effect of the covariance of $\boldsymbol{r}$ on the behaviour of the tensors $\left\langle\boldsymbol{\partial R}\left(\boldsymbol{r}, t^{\prime}-t\right)\right\rangle$ and $\left\langle\boldsymbol{\partial} \boldsymbol{\partial R}\left(\boldsymbol{r}, t^{\prime}-t\right)\right\rangle$, and only accounts for the influence of trajectories through the average statistic $\boldsymbol{r}^{0}$. Despite this, the true behaviour of these terms is well approximated by the models in terms of both the magnitude and timescale of the decorrelations. This is illustrated in Figs. 5 and 6 which compare closure approximations with exact averages. Only the distinct, nonzero components are plotted. These are consistent with predictions from the symmetry analysis. In view of equations (22), (23), only the averages $\left\langle\partial_{1} R_{11}\right\rangle,\left\langle\partial_{1} \partial_{1} R_{11}\right\rangle$ and $\left\langle\partial_{2} \partial_{2} R_{11}\right\rangle$ are required in the closure. The figures show that the proposed closures provide an acceptable level of accuracy.

Closure of the second cumulant, $\langle\langle\mathcal{H} \boldsymbol{r}\rangle\rangle$, is based upon approximation (31) for $\left\langle\boldsymbol{\Gamma}^{\prime} \boldsymbol{u}^{\prime}\right\rangle$ coupled with the gradient approximation (26). The latter, as just discussed, performs well. The performance of the former is illustrated in Fig. 7 where, again, all the distinct, non-zero components are plotted. As can be seen, reasonable agreement is obtained for all but one of the component averages: The profiles for $\left\langle\Gamma_{22}^{\prime} u_{1}^{\prime}\right\rangle$ and $\left\langle\Gamma_{12}^{\prime} u_{2}^{\prime}\right\rangle$ are noticeably dif- 


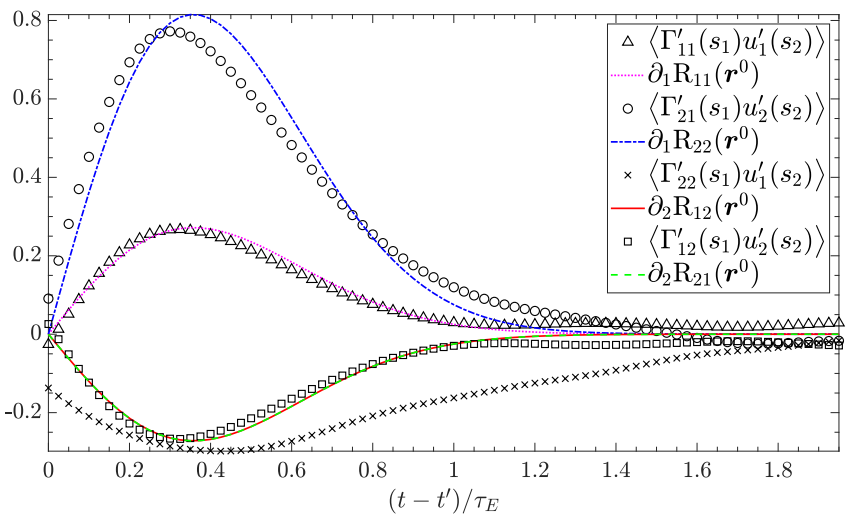

FIG. 7: (Color online) Evaluation of closure model $\left\langle\boldsymbol{\Gamma}^{\prime}\left(s_{1}\right) \boldsymbol{u}^{\prime}\left(s_{2}\right)\right\rangle \approx \boldsymbol{\partial R}\left(\boldsymbol{r}^{0}\right), S t_{E}=0.1, V_{g} / u^{\prime}=1.0$.

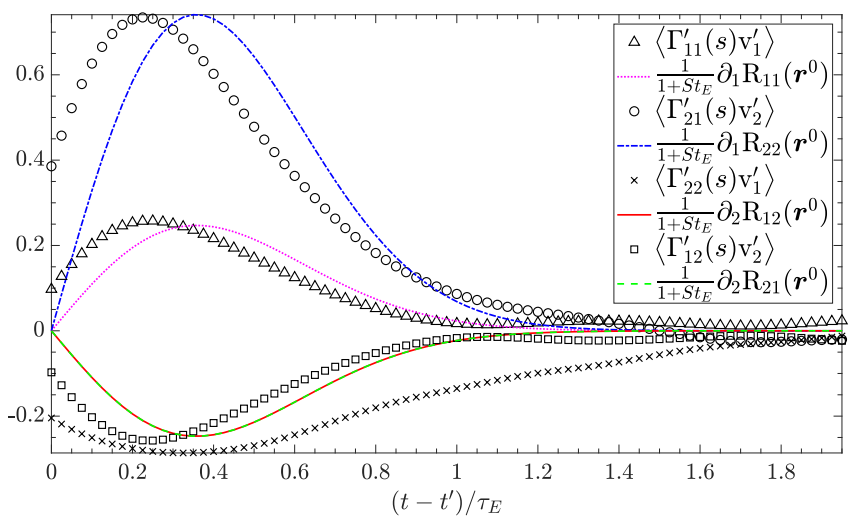

FIG. 8: (Color online) Evaluation of closure model $\left\langle\boldsymbol{\Gamma}^{\prime}(s) \mathbf{v}^{\prime}\right\rangle \approx \frac{1}{1+S t_{E}} \boldsymbol{\partial R}\left(\boldsymbol{r}^{0}\right), S t_{E}=0.1, V_{g} / u^{\prime}=1.0$.

ferent, whereas the corresponding closure approximations are identical, and fit $\left\langle\Gamma_{12}^{\prime} u_{2}^{\prime}\right\rangle$ very well. This discrepancy is a consequence of introducing the isotropic correlation tensor $\mathbf{R}$ in (31). This means that, irrespective of subsequent closure of this term, the observed anisotropy in the correlations cannot be captured. The decorrelation time-scale associated with $\left\langle\Gamma_{22}^{\prime} u_{1}^{\prime}\right\rangle$ is also seen to differ significantly from the other terms. The behaviour of this component is more characteristic $\mathcal{O}(t)$ exponential decay, rather than the $\mathcal{O}\left(t^{2}\right)$ form in the model decorrelation function $\mathrm{E}\left(s ; \tau_{L p}\right)$, which is seen to capture the other correlation profiles very well. Again this reflects the particle sampling bias in these Lagrangian correlations. The closure model for $\left\langle\boldsymbol{\Gamma}^{\prime} \mathbf{v}^{\prime}\right\rangle$, Eq. (33), is derived from that for $\left\langle\boldsymbol{\Gamma}^{\prime} \boldsymbol{u}^{\prime}\right\rangle$. Not surprisingly then, further deviations are evident between the predicted model and the true decorrelation profiles, Fig. 8. Nevertheless, on balance, the results shown in Figs. 5-8 illustrate that the underlying closures do capture essential features of the profiles. In most cases they do so very well.

The acid test of the closures based on Eqs. (31) and (26) is made by evaluating the integrand $\left\langle\mathcal{H}^{\top}: \boldsymbol{\partial R}\right\rangle$ of $\bar{\kappa}_{1}$ using these models. In principle it is possible to derive

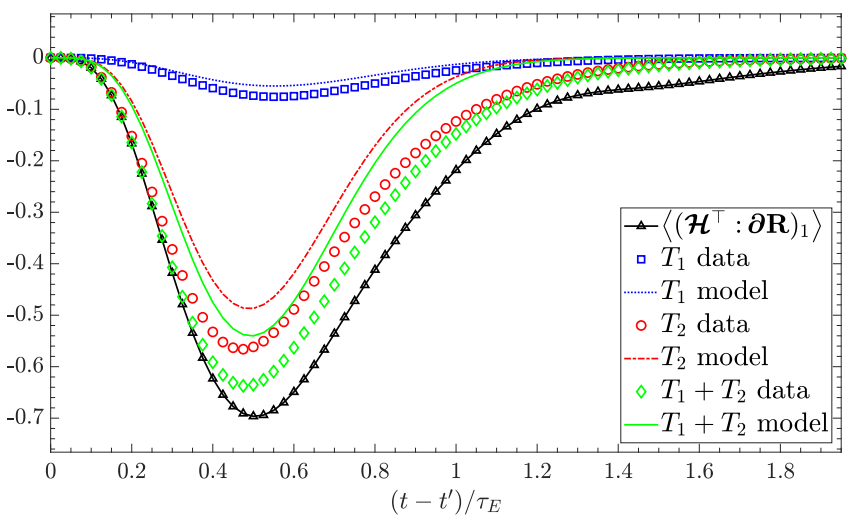

FIG. 9: (Color online) Evaluation of model for $\left\langle\left(\mathcal{H}^{\top}: \text { oR }\right)_{1}\right\rangle, S t_{E}=0.1, V_{g} / u^{\prime}=1.0$.

analytical expressions for the resulting model for this integrand. Here, however, model verification is undertaken numerically since the calculations are computationally trivial, consisting simply of a double integral in time. This is in stark contrast with the computational effort required to evaluate the unclosed expression from particle trajectory data.

The performance of the models for the terms $T_{1}$ and $T_{2}$ of the cumulant expansion (20) as evaluated using this procedure is displayed in Fig. 9. Whilst the contribution from $T_{1}$ is small in comparison to $T_{2}$, the model for $T_{1}$ is seen to accurately account for this small component. The model for $T_{2}$ is also seen to give a reasonable representation of the true behaviour, although there are discrepancies in the predicted peak value and the decorrelation timescale. The deficit in peak amplitude can be attributed to the introduction of an isotropic approximation in Eq. (31), sacrificing the true anisotropic behaviour shown in Fig. 8. The timescale discrepancy is manifest in the introduction of a single time scale, Eq. (35), invoked to model all decorrelations between fluid velocity and velocity gradient components. Despite these variations the overall model for the combined contributions of $T_{1}$ and $T_{2}$ is seen to capture important features in the time history dependence of the average $\left\langle\mathcal{H}^{\top}: \boldsymbol{\partial R}\right\rangle$. Clearly there is scope for further refinements to the detailed modelling, but these results demonstrate the potential of the proposed methodology for constructing closures for particle mass flux representations.

\section{CONCLUSIONS}

The work presented in this paper has considered a number of different representations for the particle mass flux $\rho \overline{\mathbf{v}}$, and the effectiveness of these at describing the modification in settling velocity that is exhibited by inertial particles in a uniform gravitational field. With focus on the functional correlation splitting approach in Eq. (7), an analysis of this flow configuration has high- 
lighted the symmetries that are inherent in the quantities associated with this framework. A cumulant expansion of the unclosed average in Eq. (7) has further enabled investigation into the importance of interactions involving the non-local path history effects which are sampled along particle trajectories, and a closure model has been constructed that is able to capture the essential features of this underlying behaviour.

Importantly, the functional correlation splitting approach has been shown to account for the complete effect of the increase in settling velocity that is exhibited by particles in a Gaussian flow field. Two key factors that play a role in this have been highlighted in the work: The first of these is how the the fluid velocity gradient sampled along particle trajectories influences the dynamics of the particle response tensor $\mathcal{H}$. The second is the statistical spatio-temporal structure of the turbulence as represented by the fluid velocity correlation tensor $\mathbf{R}$. Crucially it is the interaction between these two quantities that is responsible for recovering an exact representation of flux modification.

That the mass flux associated with small-scale effects such as the increase in particle settling velocity can be accurately represented by Eq. (7) is a consequence of this framework being free of spurious drift [31]. This means that the model description provided by the unclosed average in Eq. (7) does not suffer from the introduction of any artificial effects in the fluid-point limit $S t \rightarrow 0$. The phenomenon of preferential concentration is dependent upon the correlation of particle spatial distribution with the flow field structures and the functional correlation splitting approach is able to fully capture this behaviour. This work therefore serves to demonstrate that such a framework offers a conceptually sound approach for representing and modelling small-scale flux contributions which arise from preferential sampling of the flow by particles.

The other main contribution of this work is to highlight the ability of the cumulant expansion (20) to extract the necessary behaviour from the unclosed average $\left\langle\mathcal{H}^{\top}: \boldsymbol{\partial R}\right\rangle$. Previously proposed closure approaches are unable to reproduce such effects. In addition to delineating the contributions individual cumulants make to the overall behaviour, this expansion technique has the advantage of reducing unclosed expressions to stochastic quantities directly related to the underlying particle equation of motion and fluid velocity field. This provides a means to construct closures that are able to account for the characteristic physical behaviour of even small-scale fluxes, and thereby provides a fundamental representation of these phenomena.

\section{ACKNOWLEDGMENTS}

The authors acknowledge the $\mathrm{PhD}$ funding from the School of Engineering, Newcastle University, to support Dr. Stafford's research, and also the Rocket High Perfor- mance Computing service at Newcastle University.

\section{Appendix A: KS Flow Field}

The simulation results in section $\mathrm{V}$ were obtained using the technique of kinematic simulation, in which realisations of a stochastic velocity field $\mathbf{u}(\mathbf{x}, t)$ are generated. This zero-mean field is defined on a domain $[-\mathrm{L},+\mathrm{L}]^{d}$ (in $d \in\{2,3\}$ dimensions) with periodic boundary conditions, and such that the constraints of incompressibility, homogeneity, isotropy, and statistical stationarity are all satisfied. Then $\mathbf{u}(\mathbf{x}, t)$ can be expressed in terms of its Fourier series representation as

$$
\mathbf{u}(\mathbf{x}, t)=\sum_{\mathbf{k}} \mathbf{c}_{\mathbf{k}}(t) \exp [i \mathbf{k} \cdot \mathbf{x}]
$$

where $\mathbf{k}=\frac{\pi}{\mathrm{L}} \mathbf{n}=\Delta k \mathbf{n}, \mathbf{n} \in \mathbb{Z}^{d}$. The amplitude coefficients $\mathbf{c}_{\mathbf{k}}(t) \in \mathbb{C}^{3}$ are defined via $\mathbf{c}_{\mathbf{k}}=\mathbf{z}_{\mathbf{k}} \times \mathbf{k} \exp \left[i \omega_{\mathbf{k}} t\right]$, with $\mathbf{z}_{\mathbf{k}}=\frac{1}{2} \alpha_{\mathbf{k}}\left(\boldsymbol{\zeta}_{\mathbf{k}}-i \boldsymbol{\xi}_{\mathbf{k}}\right)$ and normally distributed random variables $\boldsymbol{\zeta}_{\mathbf{k}}, \boldsymbol{\xi}_{\mathbf{k}} \sim \mathrm{N}\left(\mathbf{0}, \sigma_{\mathrm{z}}^{2} \mathrm{I}\right)$, and $\omega_{\mathbf{k}} \sim \mathrm{N}\left(0, \sigma_{\omega}^{2}\right)$, subject to the constraints (to give $\mathbf{u} \in \mathbb{R}^{3}$ ), $\boldsymbol{\zeta}_{-\mathbf{k}}=-\boldsymbol{\zeta}_{\mathbf{k}}$, $\boldsymbol{\xi}_{-\mathbf{k}}=\boldsymbol{\xi}_{\mathbf{k}}, \omega_{-\mathbf{k}}=-\omega_{\mathbf{k}}$. This method of construction results in a two-point, two-time correlation tensor $\mathbf{R}(\mathbf{r}, s)$ expressible as a product of independent spatial and temporal correlation functions, $\mathbf{R}(\mathbf{r}, s)=\mathbf{Q}(\mathbf{r}) \mathrm{E}_{\omega}(s)$, with the temporal correlations following a Gaussian profile

$$
\mathrm{E}_{\omega}(s)=\left\langle\exp \left[i \omega_{\mathbf{k}} s\right]\right\rangle=\exp \left[-\frac{1}{2} \sigma_{\omega}^{2} s^{2}\right] .
$$

The parameters $\alpha_{\mathbf{k}}$ and $\sigma_{\mathrm{z}}$ may be chosen so that spatial correlations of the resulting field $\mathbf{u}$ conform to a given form of $\mathbf{Q}$. Consistent with an isotropic system this must take the form

$$
\mathrm{Q}_{i j}(\mathbf{r})={u^{\prime 2}}^{2}\left[\frac{\mathrm{r}_{i} \mathrm{r}_{j}}{\mathrm{r}^{2}}(f(\mathrm{r})-g(\mathrm{r}))+\delta_{i j} g(\mathrm{r})\right]
$$

where $\mathrm{r}=|\mathbf{r}|$, and the longitudinal and lateral decorrelation functions $f(\mathrm{r}), g(\mathrm{r})$ are related through incompressibility by

$$
g(\mathrm{r})=f(\mathrm{r})+\frac{1}{(d-1)} \mathrm{r} f^{\prime}(\mathrm{r})
$$

with $f(0)=g(0)=1$. Consistent with the standard Batchelor-Townsend energy spectrum [32, 33], the form of $f(\mathrm{r})$ is taken to be

$$
f(\mathrm{r})=\exp \left[-\frac{1}{2} \sigma_{k}^{2} \mathrm{r}^{2}\right] .
$$

To reproduce this from the simulated velocity field the parameters $\alpha_{\mathbf{k}}$ and $\sigma_{\mathrm{z}}$ are given by

$$
\begin{aligned}
\alpha_{\mathbf{k}} & =\left\{(2 \pi)^{-\frac{d}{2}}(\Delta k)^{d} \sigma_{k}^{-(d+2)} \exp \left[-\frac{1}{2} \frac{k^{2}}{\sigma_{k}^{2}}\right]\right\}^{\frac{1}{2}}, \\
\frac{1}{2} \sigma_{\mathrm{z}}^{2} & =\frac{1}{d-1} u^{\prime 2},
\end{aligned}
$$


subject to $\sigma_{k} \mathrm{~L} \gg 1$. This specification of $f(\mathrm{r})$ and $\mathrm{E}_{\omega}(s)$ also directly yields the longitudinal integral lengthscale $L_{11}$ and Eulerian integral timescale $\tau_{E}$ of the velocity field as

$$
\begin{aligned}
L_{11} & =\int_{0}^{\infty} f(\mathrm{r}) d \mathrm{r}=\sqrt{\frac{\pi}{2}} \sigma_{k}^{-1}, \\
\tau_{E} & =\int_{0}^{\infty} \mathrm{E}_{\omega}(s) d s=\sqrt{\frac{\pi}{2}} \sigma_{\omega}^{-1} .
\end{aligned}
$$

\section{Appendix B: Higher Order Derivatives of the Two-Point Correlation Tensor for Isotropic Flow}

Consistent with appendix A, and the decoupling of spatial from temporal correlations in a kinematically simulated velocity field, write $\mathbf{R}(\mathbf{r}, s)=\mathbf{Q}(\mathbf{r}) \mathrm{E}_{\omega}(s)$. Then, using Eq. (A5), the homogeneous and isotropic spatial correlation tensor $\mathbf{Q}(\mathbf{r})$ takes the form

$$
\mathrm{Q}_{j i}=\frac{u^{\prime 2}}{d-1}\left\{\sigma_{\mathrm{k}}^{2} \mathrm{r}_{j} \mathrm{r}_{i}-\left[\sigma_{\mathrm{k}}^{2} \mathrm{r}^{2}-(d-1)\right] \delta_{j i}\right\} f(\mathrm{r})
$$

To simplify the presentation of the spatial derivatives of this tensor it is convenient to introduce a sum over permutations notation. Let $\sum_{3} a_{k j i}$ denote the sum over all permutations of the 3 symbols in the triple $(k, j, i)$. That is

$$
\sum_{3} a_{k j i}=a_{k j i}+a_{k i j}+a_{j k i}+a_{j i k}+a_{i k j}+a_{i j k} .
$$

Sums over permutations of other $n$-tuples, e.g. $\sum_{4} b_{l k j i}$, are defined similarly. Then, with the interpretation that the partial derivative operator acts upon $\mathbf{r}$ in the following, we have

$$
\begin{aligned}
& \partial_{k} \mathrm{Q}_{j i}=-\frac{u^{\prime 2} \sigma_{\mathrm{k}}^{2}}{d-1}\left\{\sigma_{\mathrm{k}}^{2} \mathrm{r}_{k} \mathrm{r}_{j} \mathrm{r}_{i}-\left(\left[\sigma_{\mathrm{k}}^{2} \mathrm{r}^{2}-(d+2)\right] \mathrm{r}_{k} \delta_{j i}+\frac{1}{2} \sum_{3} \mathrm{r}_{k} \delta_{j i}\right)\right\} f(\mathrm{r}) \\
& \partial_{l} \partial_{k} \mathrm{Q}_{j i}=\frac{u^{\prime 2} \sigma_{\mathrm{k}}^{2}}{d-1}\left\{\sigma_{\mathrm{k}}^{4} \mathrm{r}_{l} \mathrm{r}_{k} \mathrm{r}_{j} \mathrm{r}_{i}-\sigma_{\mathrm{k}}^{2}\left(\left[\sigma_{\mathrm{k}}^{2} \mathrm{r}^{2}-(d+4)\right] \mathrm{r}_{l} \mathrm{r}_{k} \delta_{j i}+\frac{1}{4} \sum_{4} \mathrm{r}_{l} \mathrm{r}_{k} \delta_{j i}\right)\right. \\
& \left.+\left(\left[\sigma_{\mathrm{k}}^{2} \mathrm{r}^{2}-(d+2)\right] \delta_{l k} \delta_{j i}+\frac{1}{8} \sum_{4} \delta_{l k} \delta_{j i}\right)\right\} f(\mathrm{r}) \\
& \partial_{m} \partial_{l} \partial_{k} \mathrm{Q}_{j i}=-\frac{u^{\prime 2} \sigma_{\mathrm{k}}^{4}}{d-1}\left\{\sigma_{\mathrm{k}}^{4} \mathrm{r}_{m} \mathrm{r}_{l} \mathrm{r}_{k} \mathrm{r}_{j} \mathrm{r}_{i}-\sigma_{\mathrm{k}}^{2}\left(\left[\sigma_{\mathrm{k}}^{2} \mathrm{r}^{2}-(d+6)\right] \mathrm{r}_{m} \mathrm{r}_{l} \mathrm{r}_{k} \delta_{j i}+\frac{1}{12} \sum_{5} \mathrm{r}_{m} \mathrm{r}_{l} \mathrm{r}_{k} \delta_{j i}\right)\right. \\
& \left.+\left(\frac{1}{2}\left[\sigma_{\mathrm{k}}^{2} \mathrm{r}^{2}-(d+4)\right] \delta_{i j} \sum_{3} \mathrm{r}_{m} \delta_{l k}+\frac{1}{8} \sum_{5} \mathrm{r}_{m} \delta_{l k} \delta_{j i}\right)\right\} f(\mathrm{r}) \\
& \partial_{n} \partial_{m} \partial_{l} \partial_{k} \mathrm{Q}_{j i}=\frac{u^{\prime 2} \sigma_{\mathrm{k}}^{4}}{d-1}\left\{\sigma_{\mathrm{k}}^{6} \mathrm{r}_{n} \mathrm{r}_{m} \mathrm{r}_{l} \mathrm{r}_{k} \mathrm{r}_{j} \mathrm{r}_{i}-\sigma_{\mathrm{k}}^{4}\left(\left[\sigma_{\mathrm{k}}^{2} \mathrm{r}^{2}-(d+8)\right] \mathrm{r}_{n} \mathrm{r}_{m} \mathrm{r}_{l} \mathrm{r}_{k} \delta_{j i}+\frac{1}{48} \sum_{6} \mathrm{r}_{n} \mathrm{r}_{m} \mathrm{r}_{l} \mathrm{r}_{k} \delta_{j i}\right)\right. \\
& +\sigma_{\mathrm{k}}^{2}\left(\frac{1}{4}\left[\sigma_{k}^{2} \mathrm{r}^{2}-(d+6)\right] \delta_{j i} \sum_{4} \mathrm{r}_{n} \mathrm{r}_{m} \delta_{l k}+\frac{1}{16} \sum_{6} \mathrm{r}_{n} \mathrm{r}_{m} \delta_{l k} \delta_{j i}\right) \\
& \left.-\left(\frac{1}{8}\left[\sigma_{\mathrm{k}}^{2} \mathrm{r}^{2}-(d+4)\right] \delta_{j i} \sum_{4} \delta_{n m} \delta_{l k}+\frac{1}{48} \sum_{6} \delta_{n m} \delta_{l k} \delta_{j i}\right)\right\} f(\mathrm{r})
\end{aligned}
$$

\section{Appendix C: Correlation Splitting for Velocity Gradient Flux Closure}

The expression for $\langle\boldsymbol{\Gamma}(t)\rangle_{\mathbf{x}}$ given by Eq. (27) is obtained using the same functional correlation splitting technique that produced Eq. (7). The derivation, outlined here, makes use of the particle number density $\varrho(\mathbf{x}, t)=\delta(\boldsymbol{x}(t)-\mathbf{x})$ and ensemble-averaged number density $\rho(\mathbf{x}, t)=\langle\varrho(\mathbf{x}, t)\rangle$ introduced in section I. Recalling that $\Gamma_{i m}(t)=\partial_{m} \mathrm{u}_{i}(\boldsymbol{x}(t), t)$, the properties of $\varrho$ can be used to write

$$
\rho(\mathbf{x}, t)\left\langle\Gamma_{i m}(t)\right\rangle_{\mathbf{x}}=\left\langle\varrho(\mathbf{x}, t) \partial_{m} \mathrm{u}_{i}(\mathbf{x}, t)\right\rangle .
$$

Manipulation of the spatial derivative then yields

$$
\left\langle\varrho \partial_{m} \mathrm{u}_{i}\right\rangle=\partial_{m}\left\langle\varrho \mathrm{u}_{i}\right\rangle-\left\langle\mathrm{u}_{i} \partial_{m} \varrho\right\rangle .
$$

The first term on the right-hand side of this expression introduces $\bar{\kappa}_{i}$ again, Eq. (7). In the present context this will be spatially uniform, and therefore the gradient involved in the first term will be zero. For the second term, the same correlation splitting approach can be utilised 
$[13,15]$. For a zero-mean Gaussian velocity field $\mathbf{u}$ this results in

$$
\left\langle\mathrm{u}_{i} \partial_{m} \varrho\right\rangle=\int_{0}^{t} \int_{\mathbf{x}^{\prime}} \mathrm{R}_{i j}\left(\mathbf{x}, t ; \mathbf{x}^{\prime}, t^{\prime}\right)\left\langle\frac{\delta\left(\partial_{m} \varrho\right)}{\delta \mathrm{u}_{j}\left(\mathbf{x}^{\prime}, t^{\prime}\right) d \mathbf{x}^{\prime} d t^{\prime}}\right\rangle d \mathbf{x}^{\prime} d t^{\prime}
$$

The functional derivative in this expression can be rewritten using the chain rule as [15]

$$
\frac{\delta\left(\partial_{m} \varrho\right)}{\delta \mathrm{u}_{j}\left(\mathbf{x}^{\prime}, t^{\prime}\right) d \mathbf{x}^{\prime} d t^{\prime}}=-\delta\left(\boldsymbol{x}\left(t^{\prime}\right)-\mathbf{x}^{\prime}\right) \mathcal{H}_{k j}\left(t ; t^{\prime}\right) \partial_{k} \partial_{m} \varrho
$$

with the response tensor $\mathcal{H}\left(t ; t^{\prime}\right)$ defined, as before, by

$$
\mathcal{H}_{k j}\left(t ; t^{\prime}\right)=\frac{\delta x_{k}(t)}{\delta \mathrm{u}_{j}\left(\boldsymbol{x}\left(t^{\prime}\right), t^{\prime}\right) d t^{\prime}}
$$

Using (C4) in (C3) produces the simplification

$$
\left\langle\mathrm{u}_{i} \partial_{m} \varrho\right\rangle=-\int_{0}^{t}\left\langle\mathcal{H}_{k j}\left(t ; t^{\prime}\right) \mathrm{R}_{j i}\left(\boldsymbol{x}\left(t^{\prime}\right), t^{\prime} ; \mathbf{x}, t\right) \partial_{k} \partial_{m} \varrho\right\rangle d t^{\prime}
$$

Noting that $\mathcal{H}\left(t ; t^{\prime}\right)$ is independent of $\mathbf{x}$, manipulation of the spatial derivatives acting on $\varrho$ yields, in homogeneous systems,

$$
\left\langle\mathrm{u}_{i} \partial_{m} \varrho\right\rangle=-\int_{0}^{t}\left\langle\mathcal{H}_{k j}\left(t ; t^{\prime}\right) \partial_{m} \partial_{k} \mathrm{R}_{j i}\left(\boldsymbol{x}\left(t^{\prime}\right), t^{\prime} ; \mathbf{x}, t\right) \varrho\right\rangle d t^{\prime}
$$

Hence, using the filtering property of $\varrho$ we obtain from Eq. (C2)

$$
\left\langle\partial_{m} \mathrm{u}_{i}(t)\right\rangle_{\mathbf{x}}=\int_{0}^{t}\left\langle\mathcal{H}_{k j}\left(t ; t^{\prime}\right) \partial_{m} \partial_{k} \mathrm{R}_{j i}\left(\boldsymbol{x}\left(t^{\prime}\right), t^{\prime} ; \mathbf{x}, t\right)\right\rangle_{\mathbf{x}} d t^{\prime}
$$

This enables a closure model for $\langle\boldsymbol{\Gamma}(t)\rangle_{\mathbf{x}}$ to be constructed which is consistent with the procedure used for $\langle\varrho \mathbf{u}\rangle$ in Section IV.

\section{Appendix D: Model for the Steady-state Particle-Fluid Correlations}

The expression in Eq. (32) emanates from a PDF model for the steady-state particle-fluid correlations [26]. Specifically, a generalised Langevin model is introduced for $\mathbf{u}(\boldsymbol{x}(t), t)$, and along with Eq. (5) for the evolution of $\boldsymbol{v}(t)$, the statistics of the joint particle-fluid distribution $p(\boldsymbol{u}, \mathbf{v})$ can be derived. In particular, the distribution of $\boldsymbol{v}(t)$ conditional on $\mathbf{u}(\boldsymbol{x}(t), t)$ can be obtained by making use of the relationship $p(\mathbf{v} \mid \boldsymbol{u})=p(\boldsymbol{u}, \mathbf{v}) / p(\boldsymbol{u})$, and yields a Gaussian with mean $\hat{\mathbf{v}}(\boldsymbol{u})$ and covariance matrix $\Theta_{\mathbf{v} \mid \boldsymbol{u}}$ given by

$$
\begin{aligned}
\hat{\mathbf{v}}(\boldsymbol{u}) & =\frac{1}{1+S t_{E}} \boldsymbol{u}+\mathbf{v}_{g} \\
\boldsymbol{\Theta}_{\mathbf{v} \mid \boldsymbol{u}} & =\frac{S t_{E}}{\left(1+S t_{E}\right)^{2}} u^{\prime 2} \mathbf{I}
\end{aligned}
$$

Specification of the particle velocity in accordance with the Gaussian distribution $p(\mathbf{v} \mid \boldsymbol{u})$ is achieved using the formula $\mathbf{v}=\sqrt{\boldsymbol{\Theta}_{\mathbf{v} \mid \boldsymbol{u}}} \cdot \boldsymbol{z}+\hat{\mathbf{v}}(\boldsymbol{u})$ where $\boldsymbol{z}$ is the standard normal deviate, from which it follows that using Eqs. (D1) and (D2) results in the expression given by Eq. (32).
[1] M. R. Maxey, J. Fluid Mech. 174, 441 (1987).

[2] A. D. Bragg and L. R. Collins, New J. Phys. 16, 055013 (2014).

[3] A. D. Bragg and L. R. Collins, New J. Phys. 16, 055014 (2014).

[4] L. I. Zaichik and V. M. Alipchenkov, New J. Phys. 11, 103018 (2009).

[5] A. D. Bragg, P. J. Ireland, and L. R. Collins, J. Fluid Mech. 780, 327 (2015).

[6] R. H. A. IJzermans, M. W. Reeks, E. Meneguz, M. Picciotto, and A. Soldati, Phys. Rev. E 80, 015302(R) (2009).

[7] R. H. A. IJzermans, E. Meneguz, and M. W. Reeks, J. Fluid Mech. 653, 99 (2010).

[8] E. Meneguz and M. W. Reeks, J. Fluid Mech. 686, 338 (2011).

[9] M. Esmaily-Moghadam and A. Mani, Phys. Rev. Fluids 1, 084202 (2016).
[10] M. Esmaily and A. Mani, Phys. Rev. Fluids 5, 084303 (2020).

[11] M. W. Reeks, Phys. Fluids 3, 446 (1991).

[12] A. Bragg, D. C. Swailes, and R. Skartlien, Phys. Fluids 24, 103304 (2012).

[13] V. I. Klyatskin, Dynamics Of Stochastic Systems (Elsevier, 2005).

[14] I. V. Derevich and L. I. Zaichik, J. Appl. Math. Mech. 54, 631 (1990).

[15] D. Swailes and K. Darbyshire, Physica A 242, 38 (1997).

[16] R. V. R. Pandya and F. Mashayek, AIAA J. 41, 841 (2003).

[17] M. Reeks, D. C. Swailes, and A. D. Bragg, Phys. Rev. E 97, 023104 (2018).

[18] M. J. Beran, Statistical Continuum Theories (Wiley, New York, 1968).

[19] M. W. Reeks, arXiv:1205.2731 , 1 (2012).

[20] J. Chun, D. L. Koch, S. L. Rani, A. Ahluwalia, and L. R. Collins, J. Fluid Mech. 536, 219 (2005). 
[21] A. Aliseda, A. Cartellier, F. Hainaux, and J. C. Lasheras, J. Fluid Mech. 468, 77 (2002).

[22] P. D. Huck, C. Bateson, R. Volk, A. Cartellier, M. Bourgoin, and A. Aliseda, J. Fluid Mech. 846, 1059 (2018).

[23] D. Swailes and K. Darbyshire, Physica A 262, 307 (1999).

[24] M. W. Reeks, J. Fluid Mech. 522, 263 (2005).

[25] R. Skartlien, Int. J. Multiph. Flow 33, 1006 (2007).

[26] P. v. Dijk and D. Swailes, J. Comp. Phys. 231, 4904 (2012).

[27] L. Wang and M. Maxey, J. Fluid Mech. 256, 27 (1993).

[28] G. H. Good, P. J. Ireland, G. P. Bewley, E. Bodenschatz, L. R. Collins, and Z. Warhaft, J. Fluid Mech. 759 (2014).
[29] J. Tom and A. D. Bragg, J. Fluid Mech. 871, 244 (2019).

[30] L.-P. Wang and D. E. Stock, J. Atmos. Sci. 50, 1897 (1993).

[31] A. Bragg, D. C. Swailes, and R. Skartlien, Phys. Rev. E 86, 056306 (2012).

[32] G. K. Batchelor and A. A. Townsend, Proc. R. Soc. A 194, 527 (1948).

[33] U. Schumann and G. S. Patterson, J. Fluid Mech. 88, 685 (1978). 